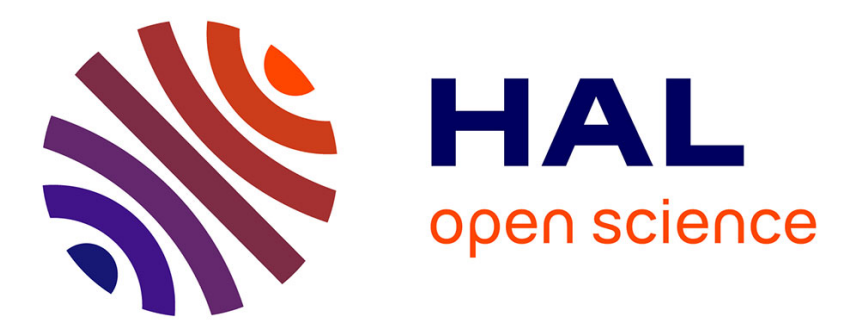

\title{
Shear-banding fluid(s) under time-dependent shear flows. Part II: A test of the Moorcroft-Fielding criteria
}

Alice Briole, Laura Casanellas, Marc-Antoine Fardin, Charlotte Py, Olivier Cardoso, Julien Browaeys, Sandra Lerouge

\section{To cite this version:}

Alice Briole, Laura Casanellas, Marc-Antoine Fardin, Charlotte Py, Olivier Cardoso, et al.. Shearbanding fluid(s) under time-dependent shear flows. Part II: A test of the Moorcroft-Fielding criteria. Journal of Rheology, 2021, 65 (6), pp.1201-1217. 10.1122/8.0000304 . hal-03345301

\author{
HAL Id: hal-03345301 \\ https://hal.science/hal-03345301
}

Submitted on 15 Sep 2021

HAL is a multi-disciplinary open access archive for the deposit and dissemination of scientific research documents, whether they are published or not. The documents may come from teaching and research institutions in France or abroad, or from public or private research centers.
L'archive ouverte pluridisciplinaire HAL, est destinée au dépôt et à la diffusion de documents scientifiques de niveau recherche, publiés ou non, émanant des établissements d'enseignement et de recherche français ou étrangers, des laboratoires publics ou privés. 


\title{
Shear-banding fluid(s) under time-dependent flows: a test of the Moorcroft-Fielding criteria (Part II)
}

\author{
Alice Briole, ${ }^{1}$ Laura Casanellas, ${ }^{2}$ Marc-Antoine Fardin, ${ }^{3}$ Charlotte Py, ${ }^{1}$ Olivier Cardoso,,${ }^{1}$ Julien Browaeys, ${ }^{1}$ \\ and Sandra Lerouge 1 \\ 1)Laboratoire Matière et Systemes Complexes, CNRS UMR 7057 Université de Paris, 10 rue Alice Domon et Léonie Duquet, \\ 75013 Paris, France \\ 2) Laboratoire Charles Coulomb, CNRS UMR 5221 Université de Montpellier, Place Eugène Bataillon, 34095 Montpellier Cedex 5, \\ France.s \\ 3) Institut Jacques Monod, CNRS UMR 7592 Université de Paris, 15 rue Hélène Brion 75013 Paris, \\ France.
}

(Dated: 28 July 2021)

Complex systems often exhibit shear banding - the coexistence of two different states characterized by their internal structuring and local shear rates. For some of them, the heterogeneous flow corresponds to the final steady state response while for others, shear banding can only be transient, the banding structure healing back to homogeneous flow in the ultimate steady state after long-lived periods. In order to explain the diversity of observations, Moorcroft and Fielding have established general criteria for the onset of banding in time-dependent flows of complex systems, ranging from polymeric fluids to soft glassy materials [Moorcroft et al., Phys. Rev. Lett., 2013, 110, 086001]. The proposed criteria are based on the time evolution of the bulk rheological response function of the system to a given time-dependent flow protocol and are associated with a specific signature in the mechanical response. In this contribution, we test the validity of these criteria in the case of two common time-dependent flow protocols: a step stress and a shear startup. Two types of fluids are examined. On the one hand, a wormlike micelles system exhibiting steady shear banding is studied experimentally, using rheometry coupled with direct visualisations and particle image velocimetry. On the other hand, we analyse previous literature on yield stress fluids exhibiting transient shear banding. Under creep flow, for both types of fluids the onset of banding arises in a time window compatible with the Moorcroft-Fielding criterion. However, the mechanical signature, i.e. the shape of the bulk mechanical signal as a function of time is not the one expected within some of the specific models with which the general Moorcroft-Fielding criteria were tested numerically. Under shear startup, both types of fluids behave differently. The criterion holds for yield stress fluids, the onset of banding arising just after the stress overshoot, as expected. On the contrary, for wormlike micelles the window of instability is delayed, even if the overshoot clearly plays a crucial role in the nucleation of the shear-induced structures. Regardless of the flow protocol or the system, wall slip seems to go hand in hand with banding indicating that it is a key ingredient to take into account.

\section{INTRODUCTION}

Shear banding is an ubiquitous phenomenon in complex fluids flows where it is usually associated with the concentration of the shear in some regions of the flow $^{33,103}$. In most common situations, shear banding results in a heterogeneous flow, in which the fluid splits into two macroscopic coexisting bands of differing local shear rates and internal mesostructures for the same shear stress. The spatial organisation of the flow corresponds to two shear bands stacked along the flow gradient direction with the interface between the bands lying in the velocity-vorticity plane.

Over the past fifteen years, shear banding has been observed in various classes of complex fluids having very different mesoscopic architectures, including polymeric fluids and soft glassy materials (SGM). Shear banding has been first reported in surfactant wormlike micelles $^{21,77,100}$, and has since been observed in lyotropic lamellar surfactant phases 101,102 , micellar systems of block copolymers solutions ${ }^{79}$, polymer solutions ${ }^{15,61}$ (even if still controversial ${ }^{73}$ ), biological fluids ${ }^{18,66}$, star polymers $^{98,99}$, emulsions ${ }^{6,91}$, suspensions ${ }^{49,82}$, colloidal gels $^{25,50}$ and microgels ${ }^{32,34}$. For exhaustive bibliography regarding experimental evidence of shear banding the reader can refer to various reviews that encompass the different classes of complex fluids ${ }^{9,13,33,43,69,78,103}$. Among these systems, not all display shear banding as ultimate steady state. Indeed shear banding has been reported to be only transient in polymer solutions ${ }^{55}$ and in various SGM including simple yield stress fluids such as soft repulsive glasses ${ }^{32,34,35}$ and some thixotropic and aging materials such as attractive colloidal gels and suspensions ${ }^{49,52,82}$. In these cases, a homogeneous flow was ultimately observed after long-lived induction periods, during which shear bands persisted.

Steady-state shear banding is usually expected when the underlying constitutive curve is non-monotonic, the latter being defined as the steady state relation between total shear stress and shear rate by enforcing homogeneity of the system. In any range of shear rate with decreasing shear stress, the homogeneous flow is linearly 
mechanically unstable ${ }^{113}$ (see Fig. 1.a). The criterion for steady shear banding writes as $d \sigma / d \dot{\gamma}<0$. Along BC, the system is linearly unstable while increasing parts $\mathrm{AB}$ and $\mathrm{BD}$ corresponds to metastable states. In practice, the steady-state flow curve $\sigma=f(\dot{\gamma})$ determined experimentally exhibits a stress plateau $\left(\sigma \equiv \sigma_{p}\right)$ above a critical shear rate $\left(\dot{\gamma} \equiv \dot{\gamma}_{1}\right)$, and the flow separates into domains bearing low $\left(\dot{\gamma} \equiv \dot{\gamma}_{l}\right)$ and high $\left(\dot{\gamma} \equiv \dot{\gamma}_{h}\right)$ local shear rates, with values respectively connected to the low $\left(\dot{\gamma} \equiv \dot{\gamma}_{1}\right)$ and high $\left(\dot{\gamma} \equiv \dot{\gamma}_{2}\right)$ boundaries of the stress plateau (Fig. 1.a). In other words, between A and D, the ultimate steady-state flow is observed to be banded. In flow cells where the stress distribution is homogeneous, the stress plateau is flat while in flows with curvature such as the Taylor-Couette (TC) flow, where the stress distribution is heterogeneous, the stress plateau presents a slight slope. For systems such as viscosity-bifurcating $\mathrm{SGM}$, the low-shear rate branch is vertical $\left(\dot{\gamma}_{1} \rightarrow 0\right)$ and steady shear banding corresponds to the coexistence of an unsheared band with a flowing band. Note that it has also been shown that flow-concentration coupling may provide an alternative mechanism by making the constitutive curve monotonic but unstable to banding both in polymeric systems ${ }^{26,27,46}$ and $\mathrm{SGM}^{11,62}$. If this description of steady shear banding in terms of the global mechanical response seems to be universal across systems, the microscopic underlying mechanisms can strongly vary from a system to another, as illustrated by the variety of models that have been derived over the past thirty years to explain the observed phenomenology.

In polymeric fluids, steady-state shear banding is predicted by microscopically grounded models such as the tube model $^{36}$ and all its modern variations ${ }^{83}$, including adaptations for (breakable) living polymers ${ }^{23}$. It is also predicted by the popular Rolie-Poly (RP) model, a simplified differential version of the tube model that incorporates various refinements taking into account additional stress relaxation mechanisms ${ }^{75}$. Very recent updates, i.e. extension of the simplified tube approximation for rapid-breaking micelles ${ }^{93,94}$ and the living Rolie-Poly-fitted model ${ }^{94}$ have been proposed to predict the linear and nonlinear rheology beyond the fast breaking limit, for which the breaking/recombination time is much smaller than the reptation time. The non-monotonic character of the constitutive relation can also be tuned in simpler phenomenological models such as, among others, the diffusive Johnson-Segalman ( $d$ JS) model ${ }^{63,90}$ and the diffusive Giesekus (d-Giesekus) model ${ }^{42,51}$, which can qualitatively reproduce many of the macroscopic aspects of shear banding. Basic features of shear banding are also predicted by the Bautista-Manero-Puig model, which couples the upperconvected Maxwell constitutive equation to a kinetic equation that takes into account shear-induced structural changes ${ }^{4,5}$. Many properties of the shear-banding flow are also reproduced by models that incorporate concentration coupling with shear banding, such as the two (a)

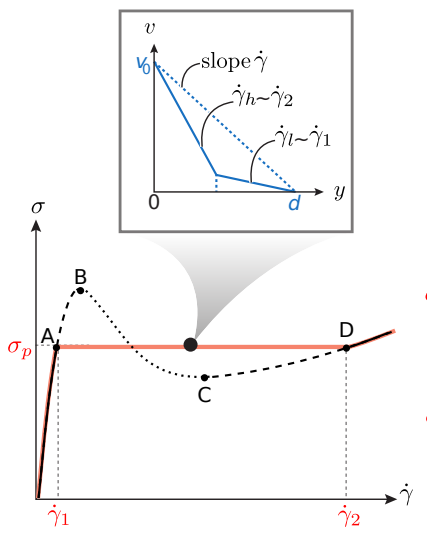

(b)

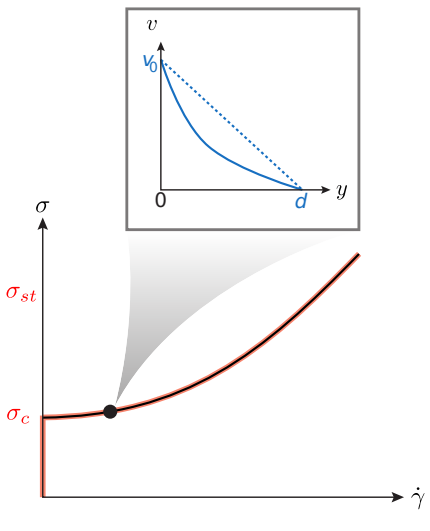

FIG. 1. Steady-state shear stress vs global shear rate in absence of flow/structure coupling and/or curvature of the flow geometry : the underlying constitutive curve is represented in black while the measured flow curve is in orange. The insets display the corresponding steady-state velocity profiles (blue straight line). In (a), the constitutive curve is non-monotonic and the corresponding measured flow curve exhibits a stress plateau extending from $\dot{\gamma}_{1}$ to $\dot{\gamma}_{2}$, a picture that presents a formal analogy with the liquid-gas transition at thermodynamical equilibrium. The black straight lines correspond to stable branches. The decreasing dotted part $(\mathrm{BC})$ is linearly unstable. The increasing dashed parts ( $\mathrm{AB}$ and $\mathrm{CD})$ correspond to metastable states. In (b), the experimental flow curve and the corresponding constitutive curve are monotonic and superimposed. In this sketch, a yield stress material that follows a Herschel-Bulkley rheology is displayed.

species Vasquez-Cook-McKinley model adapted to describe living polymers ${ }^{96,108,114,115}$

In soft glassy materials, non-monotonicity of the constitutive relation and subsequent steady-state shear banding can be captured by various types of models. The formation of permanent shear bands can be accounted for by an extended version of the 'soft glassy rheology' model that involves coupling to a relaxation-diffusion dynamics for the effective temperature ${ }^{45}$. It can also be reproduced by various fluidity models, where, in general, a local rheological model is coupled with a non-local diffusion equation governing the behaviour of a fluidity parameter usually defined as the ratio between the shear rate and the shear stress and that can be related to the rate of plastic events $8,12,25,43,48,95,111$. The nonlocal nature of the fluidity leads to cooperative effects characterized by a spatial cooperativity length scale : localized plastic events induce long-range elastic modifications of the stress over the system. Recent updates have shown that appropriate combination of mechanical noise and cooperative effects can lead to permanent banding. Mesoscopic approaches based on elastoplastic models are also capable of predicting permanent shear bands $80,81,88$. For exhaustive bibliography regarding shear-banding modeling, the reader can also refer to several reviews ${ }^{13,24,43,71,88,89}$. 
In the absence of flow-concentration coupling, systems with monotonic flow curve are not expected to admit permanent banding (see Fig. 1.b) but are likely to develop transient banding whose lifespan can extend over very long time scales, much longer than the typical time scale of the flow $\dot{\gamma}^{-1}$. The lifespan of the transients has been reported to decay as a power law of the control parameter with exponent specific to the imposed variable $(\dot{\gamma} \text { or } \sigma)^{32}$. Observation of transient shear banding in both polymer solutions and simple yield stress fluids has motivated numerous theoretical contributions over the past ten years to provide a rationale to the long-lasting heterogeneous flows in fluids that do not support banding as ultimate steady-state $1,2,7,43,44,54,59,60,76,85-87,107$. For SGM, Benzi et al. have recently shown in the framework of fluidity models that, in conditions of 'weak' noise, cooperative effects can capture long-lasting transient heterogeneous flows in quantitative agreement with experimental observations ${ }^{7}$. Another approach has suggested that shear banding can arise transiently in flows with strong time-dependence, even in fluids for which the steadily flowing state is unbanded. In this context, Moorcroft and Fielding have derived general mechanical criteria to predict the onset of transient or permanent shear banding in the inertialess limit of creeping flow. The criteria encompass most of the widely used models for the rheology of complex fluids (including polymeric and soft glassy materials $)^{85-87}$. For each time-dependent protocol such as shear startup, step stress and step strain, an analytical criterion, independent of any constitutive relation, was derived using linear stability analysis of a base state characterized by a response function calculated by enforcing homogeneous flow. Even though the criteria do not include flow-concentration coupling, the framework remains general, at least for step stress protocols, and with some caveats in shear startup, as discussed in Ref. ${ }^{44}$ and can in principle apply to systems exhibiting shear banding or not in steady state. Using numerical simulations of specific models [RP and soft glassy rheology (SGR) models], each criterion for onset of banding can be expressed in terms of a specific signature in the measured time-dependent rheological response function of the system (see ${ }^{44}$ for a synthetic road map).

For step stress protocol, starting from a fluid initially at rest and jumping the shear stress to a given value $\sigma$, the criterion for onset of banding was found to obey:

$$
K_{\sigma} \equiv \frac{d^{2} \dot{\gamma}}{d t^{2}} / \frac{d \dot{\gamma}}{d t}>0
$$

The onset of banding is then likely to occur as the shear rate temporal evolution exhibits simultaneously upward slope and upward curvature. The shape of the typical response function expressed in term of time-differential of the creep curve $\dot{\gamma}(t)$ obtained from simulations for both RP and SGR models is illustrated in Fig. 2.a where the dotted line represents the region where criterion (1) is fulfilled. Note that the shear rate response sketched in (a)

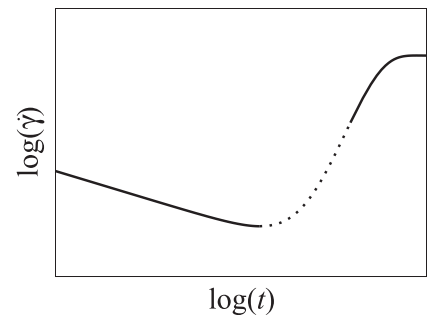

(b)

FIG. 2. (a) Sketch of the typical evolution of the global shear rate following step stress. Along the dotted part the system is likely to be linearly unstable to banding. (b) Sketch of the typical evolution of the global shear stress following step shear rate. The dotted part corresponds to the region of linear instability to banding. For systems exhibiting steady-state shear banding, the dotted part also extends over the long times. Reprinted with permission from Fielding ${ }^{4}$, copyright by the Society of Rheology.

Fig. 2.a has been computed in planar shear flow (without curvature) and without considering flow/concentration coupling, so that it is expected for systems that remain unbanded at steady state. In other words, the MoorcroftFielding criterion under step stress protocol was not examined in the simulations for the case of steady shearbanding.

For shear startup protocol, starting from an initially undisturbed sample and jumping the shear rate to a fixed value, the criterion for onset of banding was found to be:

$$
K_{\dot{\gamma}} \equiv \frac{d \sigma}{d \gamma}<0
$$

The typical temporal stress response is sketched in Fig. 2.b. It is dominated by a stress overshoot for $\gamma \sim 1$ that relaxes towards steady state. The onset of banding is supposed to occur just after the stress overshoot and to be triggered by the stress drop. Note that the most general form of equation 2 contains a correction term that involves curvature of the stress signal $\left(d^{2} \sigma / d \gamma^{2}\right)$ leading to a shift of the instability onset very slightly before the stress overshoot ${ }^{86}$. In contrast to Eqn (1), the general status of the shear startup criterion remains unclear due to the limitation of the number of dynamical viscoelastic variables allowed in the constitutive model (see Ref. ${ }^{44}$ for discussion about caveats).

In the first part of this work ${ }^{20}$, we studied the full spatio-temporal dynamics of the shear-banding flow of a surfactant wormlike micelles system following creep flow and flow start-up. We mainly focused on the long time dynamics, and especially on the development, on top of the shear-banding flow, of secondary flows triggered by elasticity. In the present study, we used the same surfactant wormlike micelles system, well-known to exhibit shear banding as ultimate steady state to test the validity of the suggested criteria. We mainly focus on the creep flow response to step stress but we also explore 
the validity of the Moorcroft-Fielding criterion in shear startup [Eqn (2)] and suggest conclusions in this flow configuration by also building on the existing exhaustive literature. Finally, we elaborate on a comparison with transient behaviors observed in simple yield stress fluids, well known to only exhibit transient banding, the ultimate steady state being homogeneous. The choice of these two types of systems allows us to encompass two possible situations observed experimentally, namely, the occurrence of banding, which will persist at steady state (case of wormlike micelles for which the constitutive curve is non-monotonic) and the occurence of banding, which will heal back to homogeneous steady-state flow (case of carbopol microgel, for which the constitutive curve is monotonic).

\section{EXPERIMENTAL DETAILS}

The complete description of the materials, their viscoelastic properties and their steady-state behaviour is given in the first part of this work ${ }^{20}$. Here, we limit ourselves to recalling the essential elements.

The experiments were conducted on a semi-dilute aqueous mixture of $0.3 \mathrm{M}$ cetyltrimethylammonium bromide $(\mathrm{CTAB})$ and $0.4 \mathrm{M}$ sodium nitrate $\left(\mathrm{NaNO}_{3}\right)$ at a temperature fixed to $28.0 \pm 0.1^{\circ} \mathrm{C}$. This system has been extensively studied over the past fifteen years and is well-known to exhibit shear banding as ultimate steady state $^{41,68,70}$. Its viscoelastic properties were found to be quite well described by the single mode Maxwell model with characteristic visco-elastic time $\tau=0.24 \pm 0.05 \mathrm{~s}$ and elastic modulus $G_{0}=243 \pm 5 \mathrm{~Pa}$. Its steady-state flow curve is characterized by a stress plateau at $\sigma \equiv \sigma_{p} \simeq$ $155 \pm 3 \mathrm{~Pa}$ indicative of gradient shear banding ${ }^{104,105}$. The stress plateau extends between two critical shear rates $\dot{\gamma} \equiv \dot{\gamma}_{1} \simeq 4.5 \pm 0.5 \mathrm{~s}^{-1}$ and $\dot{\gamma} \equiv \dot{\gamma}_{2} \simeq 73 \pm 5 \mathrm{~s}^{-1}$ and was observed to present a slope, partly due to the intrinsic curvature of the TC flow geometry. Secondary flows ${ }^{70}$ and flow-concentration coupling ${ }^{46}$ might also contribute to the slope. Along the stress plateau, the steady-state velocity profiles were shown to exhibit the expected shape for a shear-banding system, with two coexisting regions of different well-defined local shear rates $^{20}$. Slip was observed at the moving wall, a feature, which is omnipresent in shear-banding wormlike micelles ${ }^{39,72}$. We will see in the following (section III C) that slip appears as the shear-induced structures grow at the inner wall, suggesting that it results from specific interactions between the shear-induced structures and the wall, as already observed in experiments conducted in strain-controlled mode ${ }^{39}$.

In the present study the $\mathrm{CTAB} / \mathrm{NaNO}_{3}$ system was investigated through global rheological measurements, optical visualisations and velocimetry measurements, all performed in the same home-made TC flow geometry. The TC cell, which consisted in a glass outer cylinder with $25 \mathrm{~mm}$ radius and a black Delrinßinner cylinder with $24 \mathrm{~mm}$ radius and $40 \mathrm{~mm}$ height, giving a curvature ratio $\Lambda \equiv d / R i=0.04$ and an aspect ratio $\Gamma \equiv h / d=40$ was coupled to a stress-controlled rheometer (Anton Paar MCR 301), except in section III B where comparisons with data obtained with a TA Instruments ARES strain-controlled rheometer are also displayed. To determine the onset of shear banding, time-resolved direct optical visualisations and particle image velocimetry measurements were performed simultaneously with recording of the global mechanical signals. In the semidilute range, shear-banding wormlike micelles are prone to exhibit slightly turbid shear-induced structures, generating a very good optical contrast between the two bands and providing a way to finely track the interface between bands in the velocity gradient-vorticity plane $[(r, z)$ plane]. The latter was illuminated with a radial laser sheet and the intensity scattered by the sample at $90^{\circ}$ was recorded at 6 frames per second by a CCD array (AVT Stingray $1600 \times 1200$ pixels) equipped with a magnification device (Zoom 6000 Navitar with a $1.5 \times$ objective and $2 \times$ adapter tube) providing a spatial resolution along the radial direction reaching 520 pixels $/ \mathrm{mm}$. Particle image velocimetry experiments were performed by seeding the sample with $100 \mathrm{ppm}$ of tracer particles with a mean diameter of $5 \mu \mathrm{m}$ [Polyamide Seeding Particles (PSP), Dantec Dynamics]. We used a commercial 1D system from Dantec Dynamics involving a Nd:YAG pulsed laser (DualPower 66-15 Laser 2x65 mJ, $\lambda=532 \mathrm{~nm}$ ) but the velocity profiles $v(r) \equiv v_{\theta}(r)$ were computed from a home-made Python routine, which involves the 'Scikitimage' library for image processing ${ }^{109}$. Pairs of images of the $(\theta, r)$ plane were captured with a zoom-microscope lens (Zoom 6000 Navitar with a $1.5 \times$ objective and $2 \times$ adapter tube) mounted on a CCD array (FlowSense EO $4 \mathrm{M}$ ) working at an image acquisition rate of $10 \mathrm{~Hz}$. The delay between laser pulses was set to $700 \mu$ s. To take into account the curvature inherent to the TC device, each image was first unbent using piecewise affine transformation defined by the appropriate displacement of 10 by 10 control points regularly distributed along the $\theta$ and $r$ directions. The displacement between two frames was then obtained by correlation over windows of 63 pixels height along the velocity gradient direction and 2008 pixels width along the azimuthal direction. Such a large width of interrogation window was particularly useful when computing instantaneous velocity profiles since it allowed us to average along the azimuthal direction and improves the signal to noise ratio.

\section{RESULTS AND DISCUSSION}

\section{A. Test of the Moorcroft-Fielding shear-banding criterion under creep flow}

In this section, we focus on the short-time response of the system under creep flow, the ultimate goal be- 
ing to determine whether the Moorcroft-Fielding shearbanding criterion is valid or not in shear-banding wormlike micelles systems. In absence of curvature and/or flow-concentration coupling, shear-banding fluids exhibit a flow curve with a flat plateau, precluding exploration of the banding regime under imposed stress. Here we take advantage of the curved flow geometry to explore the onset of banding under creep flow of wormlike micelles. Global and local variables were measured with increased time resolution in order to provide the best correlation as possible.

\section{Early stages of the shear rate response.}

Figure 3 displays the early stages of a typical shear rate response following step stress applied from $t=0$ on a sample initially at rest with stress target inside the plateau regime $(\sigma=179 \mathrm{~Pa})$. The initial response $(t \lesssim 0.5 \mathrm{~s})$ is dominated by inertio-elastic ringing due to the coupling between the sample elasticity and the instrument rotational inertia 3,37,38,112. A quantitative description of the creep-ringing response is given in the first part of this work ${ }^{20}$.

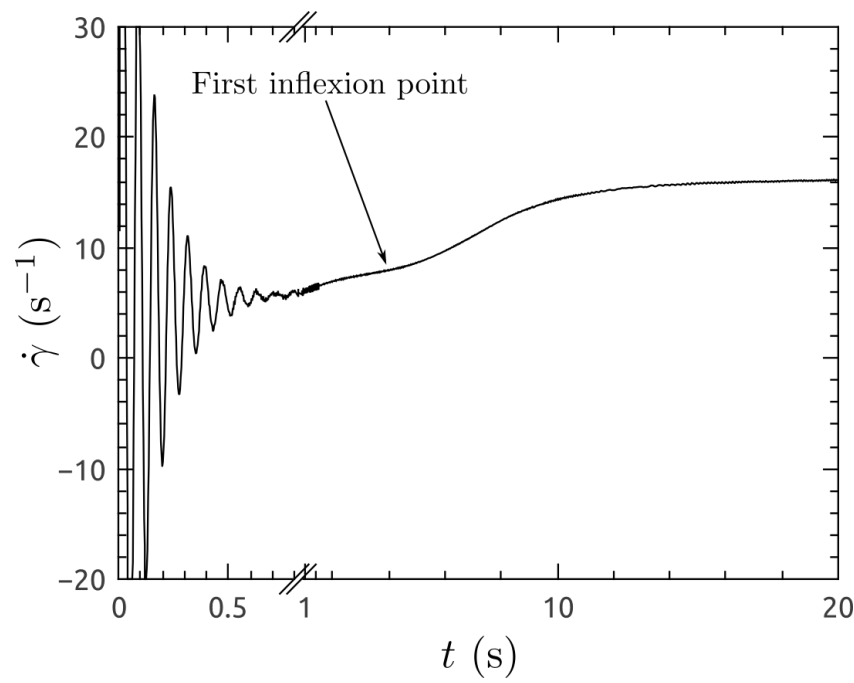

FIG. 3. Short-time response of the shear rate following step stress $\sigma=179 \mathrm{~Pa}$ applied at $t=0$ to a sample initially at rest.

The inertio-elastic ringing regime is followed by a stage, called induction period 56,57 , where the global shear rate slightly increases and, which ends at the first inflexion point in the $\dot{\gamma}(t)$ curve (Fig. 3). Beyond this point, $\dot{\gamma}$ increases with $t$ and progressively approaches its steady-state value.

The transient evolution of the shear rate can be compared with the general evolution sketched in Fig. 2.a. In our experiments the initial slow 'solid-like' creep regime produced in the simulations of the RP model is not accessible since it is most likely hidden by creep ringing. Note that inertial effects have been neglected in the nu- merical simulations so that creep ringing could not be reproduced ${ }^{87}$. Furthermore, it can also be noticed that the subsequent growth of $\dot{\gamma}$ towards its asymptotic value is slightly different from the predicted shape. Interestingly, during this transient growth, the $\dot{\gamma}(t)$ curve obviously slopes upward but the curvature changes from negative to positive at the first inflexion point indicating that criterion (1) is fulfilled right after the inflexion point of the differentiated creep curve.

In the next section, we focus on the short-time evolution of the scattered intensity and the velocity field to get information at local scale.

\section{Onset of shear banding.}

Time-resolved direct visualisations and velocimetry experiments were performed in order to detect the nucleation of the shear-banding structure as accurately as possible. From a typical time sequence as displayed in Fig. 4.a, the intensity $I(r, z)$ scattered by the sample was averaged over $z$ for each time step and a spatio-temporal diagram $\langle I(r)\rangle_{z}(t)$ was built (see Fig. 5.a). The radial intensity distribution as a function of time can reflect the organisation of the flow: in principle, we can discriminate between banding or not and the intensity profile can provide indications about the position and the apparent width of the interface profile. The latter can be diffuse and large (usually at the beginning of the band-

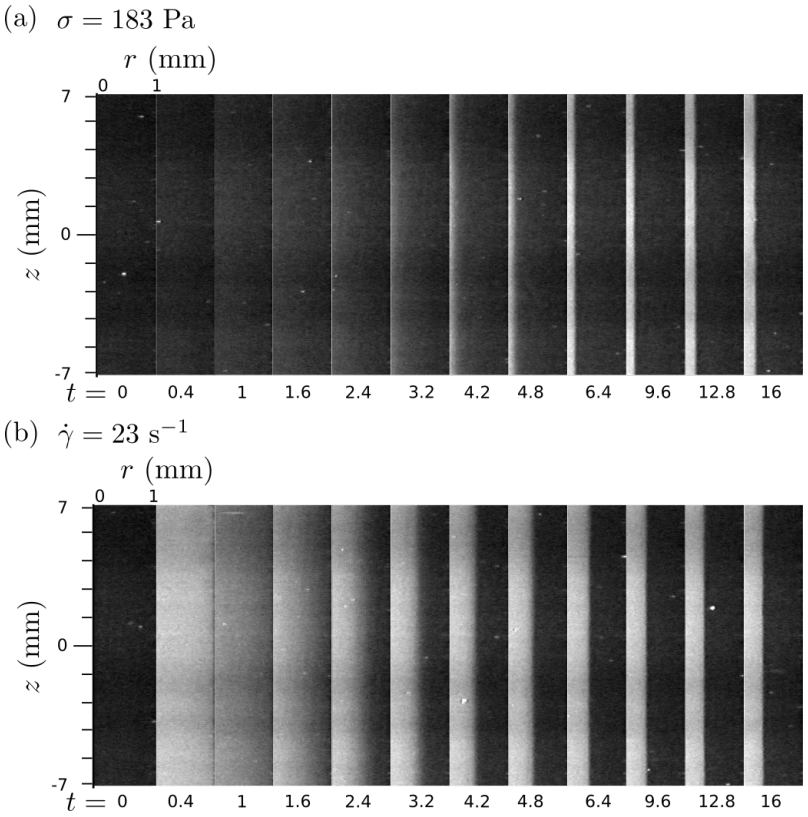

FIG. 4. Views of the $(r, z)$ plane of the TC illuminated with a radial laser sheet. Early stages of the response to (a) a step stress $\sigma=183 \mathrm{~Pa}$ and (b) a step shear rate $\dot{\gamma}=23 \mathrm{~s}^{-1}$. Note that these two values of the control parameter correspond to the same asymptotic state. The grey levels code for the intensity $I(r, z, t)$ scattered by the sample. 
ing process), sharp (when approaching steady state) or blurred (when the interface is wavy ${ }^{20}$ ). Fig. 5.a displays $\langle I(r)\rangle_{z}(t)$ for an applied stress $\sigma=183 \mathrm{~Pa}$. When focusing on the evolution of the front between dark and bright zones as a function of time, we can successively distinguish a slight increase of the intensity level in the whole gap, the growth of the intensity level closer to the inner cylinder, the building of a diffuse front and the progressive stiffening of the front followed by blurring due to undulations of the interface.

The early stages including the progressive stiffening of the front are illustrated in Fig. 5.e where we have reported the time evolution of the intensity profile over the first $6.2 \mathrm{~s}$ (delimited by the green rectangle in Fig 5.a). The main difficulty in the accurate experimental determination of the onset of banding is to define a criterion as objective as possible. Moorcroft-Fielding criteria resulting from a linear stability analysis, they apply to the very early time dynamics when heterogeneous fluctuations are first growing. Accordingly, we chose to define the onset of banding as the intensity profile departs significantly from a linear shape, that is, once curvature in the profile appears, indicating formation of a diffuse front between two zones of differing intensities and therefore differing microstructures. For $\sigma=183 \mathrm{~Pa}$ this specific 'instant' is likely to occur between 2 and $2.6 \mathrm{~s}$, as denoted by the dashed blue curves in Fig. 5.e. In practice the departure of the intensity profile from the linear shape can be well-captured using image processing. We performed image thresholding using the Renyi entropy method proposed in ImageJ software ${ }^{64}$.

Illustration of automatic thresholding with this method is given in Figs. 5.b and c, which focus on a portion of the gap over the first $24 \mathrm{~s}$ (red rectangle in Fig. 5.a). The edge detection of the thresholded image (Fig. 5.c) provides both the onset of banding as defined above and an estimate of the position of the front as a function of time (Fig. 5.d). Another possible indicator of emerging heterogeneities is given by the quantity $S_{I}(t)=\sqrt{\frac{\sum_{i}\left(\left\langle I\left(r_{i}, t\right)\right\rangle_{z}-I_{\text {lin }}\left(r_{i}, t\right)\right)^{2}}{N}}$, which represents the square root of the squared deviation from the linear intensity profile $I_{\text {lin }}$.

The onset of banding was also tracked using timeresolved PIV velocimetry. Instantaneous velocity profiles were recorded as a function of time during a short period following imposition of the shear stress (Fig. 6). As illustrated in Figs. 6 [see (a), (b) and (e).top-left panel for instance] the velocity profiles are found to be initially approximately linear.

The first signs of deviation from the linear profiles occur between $t \simeq 2$ and $3 \mathrm{~s}$ as illustrated in Fig. 6.e.topright panel. The banding structure then progressively develops. To estimate the onset of banding from velocimetry experiments, each velocity profile was fitted by a linear function and, for each of them, the square root $S_{v}$ of the mean of the squared residuals was com- puted $\left(S_{v}(t)=\sqrt{\frac{\sum_{i}\left(v\left(r_{i}, t\right)-v_{l i n}\left(r_{i}, t\right)\right)^{2}}{N}}\right)$

The whole set of results is gathered in Fig. 7 for various imposed shear stresses. The short-time shear rate response, its smoothed version and its first derivative, both computed using Savitzky-Golay filter, are represented. We also plotted the time evolution of the front obtained from the thresholding of $\langle I(r)\rangle_{z}$.

The apparent width $w$ of the front computed by fit-

$$
\sigma=183 \mathrm{~Pa}
$$

(a)

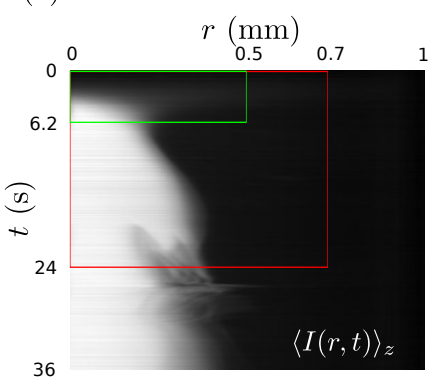

(b)

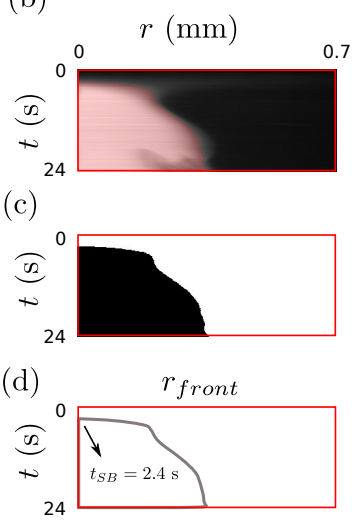

(e)

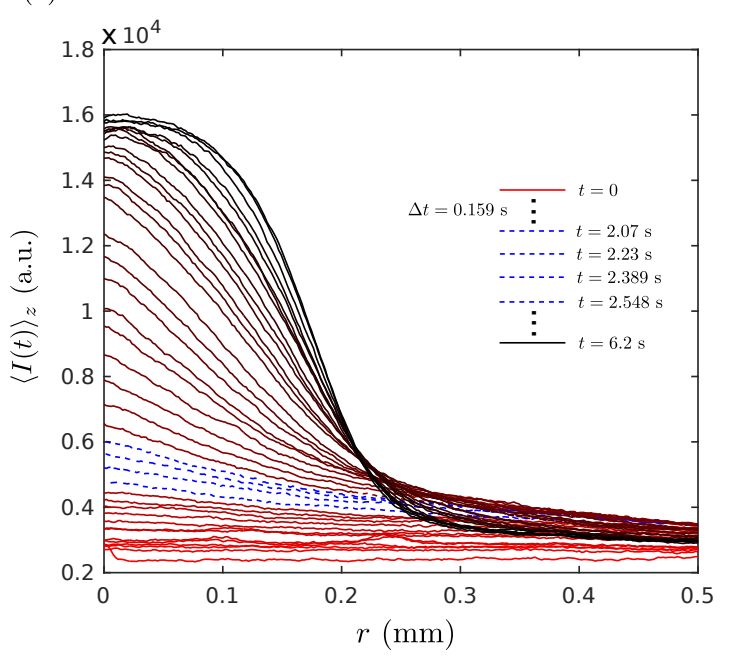

FIG. 5. Procedure used to determine the onset of banding from direct visualisations in the $(r, z)$ plane. The illustration is given for an imposed stress $\sigma=183 \mathrm{~Pa}$. (a) Spatio-temporal diagram $\langle I(r)\rangle_{z}(t)$ constructed from a time sequence as given in Fig. 4.a. For each time step, the intensity scattered by the sample is simply averaged over $z$ and the resulting intensity profile $\langle I(r)\rangle_{z}$ is then re-plotted in grey levels as a function of the time $t$. (b) Tracking of the front between bands using Renyi entropy thresholding method. Only a fraction of the gap over $24 \mathrm{~s}$ [red rectangle in (a)] is displayed. (c) Resulting black and white thresholded picture. (d) Edge detection of the thresholded image (c) providing both the onset of banding and an estimate of the front position as a function of time. (e) Temporal evolution of the intensity profile over the first $6.2 \mathrm{~s}$ [green rectangle in (a)]. The blue color is used to denote the profiles which exhibit the first noticeable signs of curvature. 
(a) $\sigma=175 \mathrm{~Pa}$

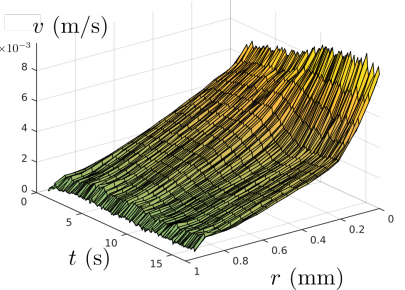

(c) $\sigma=183 \mathrm{~Pa}$

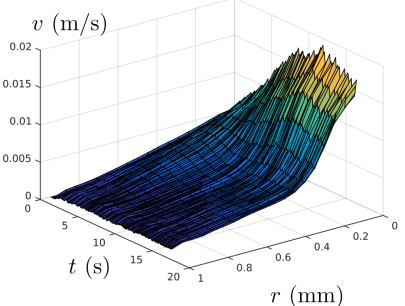

$r(\mathrm{~mm})$

(e) $\sigma=183 \mathrm{~Pa}$
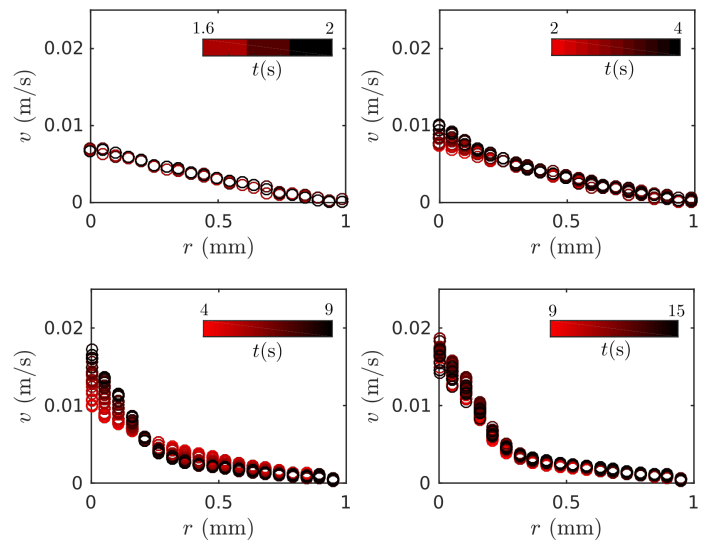

FIG. 6. Representation as a surface plot of the instantaneous velocity profiles $v(r)$ as a function of time measured using PIV technique and recorded during a short period following imposition of the shear stress (a) $\sigma=175 \mathrm{~Pa}$, (b) $\sigma=179 \mathrm{~Pa}$, (c) $\sigma=183 \mathrm{~Pa}$, (d) $\sigma=188 \mathrm{~Pa}$. (e) Selective choice of velocity profiles over successive temporal ranges for $\sigma=183 \mathrm{~Pa}$. As time evolves, the color code goes from red to black. The time interval between each represented velocity profile is $0.2 \mathrm{~s}$.

ting the intensity profile with an error function $\left[\langle I(r)\rangle_{z}=\right.$ $A * \operatorname{erf}\left(\left(r-r_{\text {front }}\right) / w\right)+I_{0}$ where $A$ is the amplitude and $I_{0}$ the intensity base line] is also displayed as a function of time. Finally, the time evolution of $S_{v}$ and $S_{I}$, the square root of the mean of the squared residual resulting from the fitting procedure of the velocity and the intensity profiles are also shown. Let us discuss the example at $\sigma=183 \mathrm{~Pa}$ (Fig. 7.c). During the shear rate growth following creep ringing, two successive changes of curvature are observed as illustrated by the local minimum and maximum in the first derivative $d \dot{\gamma} / d t$. In between these local extrema $(2.3 \leq t \leq 5.5 \mathrm{~s})$, Eqn (1) is satisfied. It turns out that, around the specific time associated with the local minimum of $d \dot{\gamma} / d t(t \simeq 2.4 \mathrm{~s})$, the intensity profile starts exhibiting slight curvature indicating growth of shear-induced structures from the inner moving wall. $S_{I}$ starts growing out of initial noise from $t \simeq 2.1 \mathrm{~s}$ (closed pink squares) while a front between a turbid zone and a clear zone clearly emerges from $t \simeq 2.4 \mathrm{~s}$ (open red circles). At the same time, the apparent front width $w$ (open blue triangles), which initially diverges starts getting finite values but still larger than the gap size indicating that the front between bands is very diffuse. The velocity profiles, which were linear for $t \leq 2 \mathrm{~s}$ (Fig. 6.e.top-left panel) become curved close to the inner wall (Fig. 6.e.topright panel). Between 2 and $4 \mathrm{~s}, S_{v}$ starts increasing out of the intrinsic noise while $w$ exhibits an abrupt decay to a value of about $150 \mu \mathrm{m}$ that reflects a rapid stiffening of the front. The subsequent stage, between 4 and $8 \mathrm{~s}$ is associated with a slow stiffening of the front, which is approaching its final (sharp) shape $(w \rightarrow 50 \mu \mathrm{m})$, and with a significant increase of $S_{v}$ related to the progressive formation of a distinct kink in the velocity profiles. The local maximum in the first derivative of $\dot{\gamma}(t)$ might be connected to this process. Note that the evolutions of $S_{I}$ and $S_{v}$ do not have to be over-interpreted since these indicators are very sensitive to noise. In PIV experiments, the intrinsic noise of the measurement is evaluated as a fraction (commonly chosen as 1/10) of pixel per time interval as long as the particles displacement remain small, i.e. inside the interrogation window. In our experiments, the pixel size is $1.7 \mu \mathrm{m}$ and the time interval between images is $700 \mu \mathrm{s}$, giving an order of magnitude of $0.3 \mathrm{~mm} / \mathrm{s}$ for the intrinsic noise of the PIV experiment. Only the variations of $S_{v}$ above this value can reasonably be interpreted as hint of emerging nonlinearity in the velocity profiles. If the growth of $S_{v}$ can reasonably be estimated (between 2 and $2.5 \mathrm{~s}$ at $183 \mathrm{~Pa}$ ), any attempt of quantitative determination of a linear growth rate is precluded. However, we can qualitatively notice that the growth rate of $S_{I}$ and $S_{v}$ are very different. This observation might be rationalized by arguing that the scattered intensity is most likely related to the polymeric stress field, which depends in a strongly nonlinear way on the velocity field.

Overall, when Eqn (1) is fulfilled in the shear rate temporal evolution, shear-induced structures start growing from the inner wall and the corresponding velocity profiles depart from the linear shape, exhibiting curvature closer to the inner wall. The situation is less clear for the lowest applied shear stress (175 Pa), for which emergence of heterogeneity in the velocity profiles seems to be slightly delayed with respect to the first inflexion point in $\dot{\gamma}(t)$. Furthermore the appearance of a distinct kink in the velocity profiles (often used as an argument to ascertain the existence of shear banding) and, equivalently, of a 'structural interface' with a sharp steady profile is observed after the window of instability given by Eqn (1), which is not unexpected since Moorcroft-Fielding criteria were derived from linear stability analysis and may not be valid when the velocity profiles become nonlinear enough.

Our observations are compatible with the results of ve- 
(a)

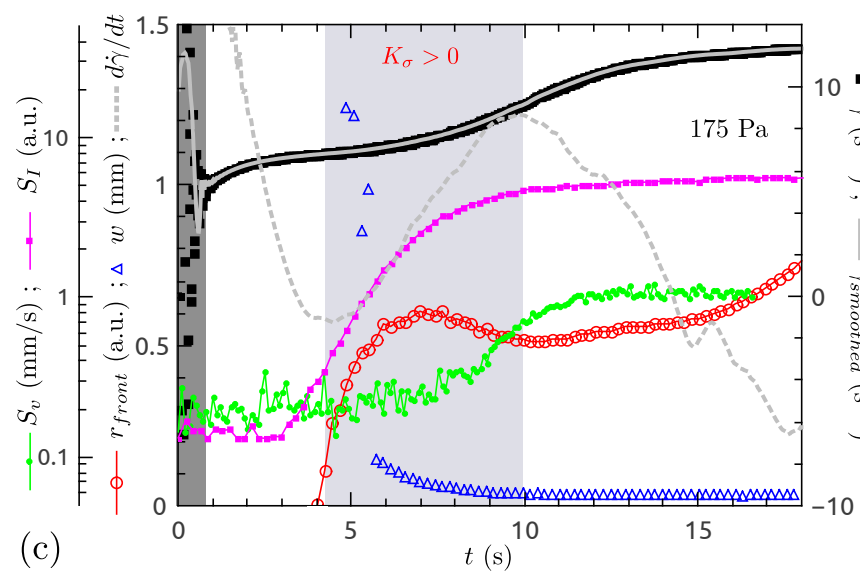

(c)

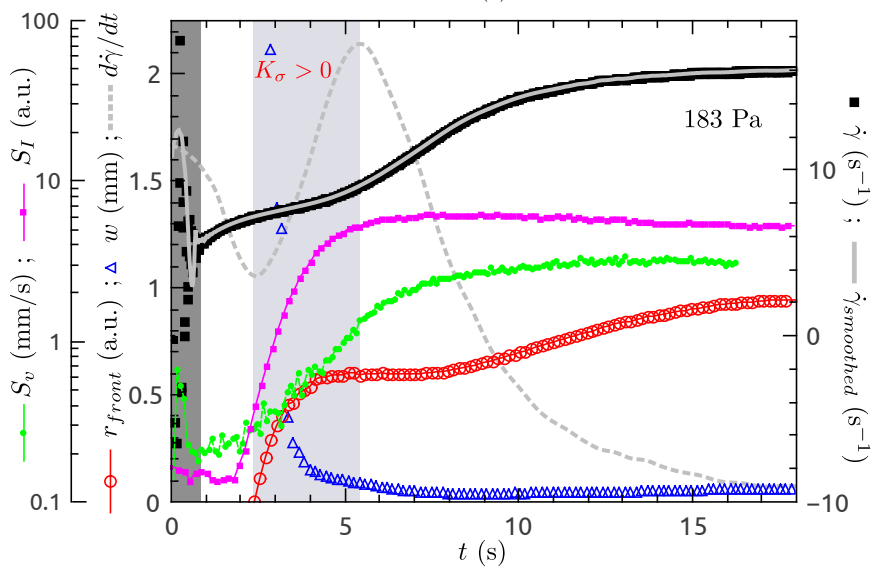

(b)

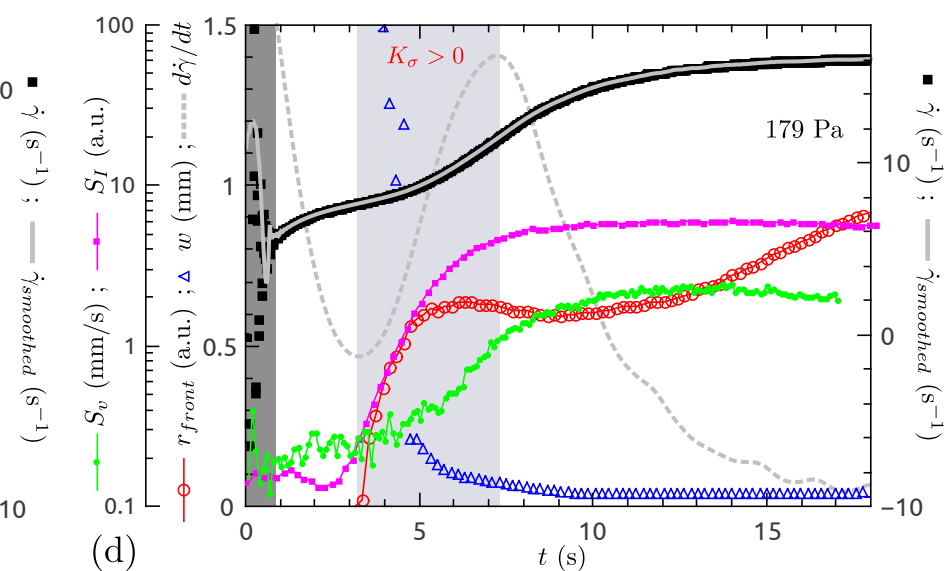

(d)

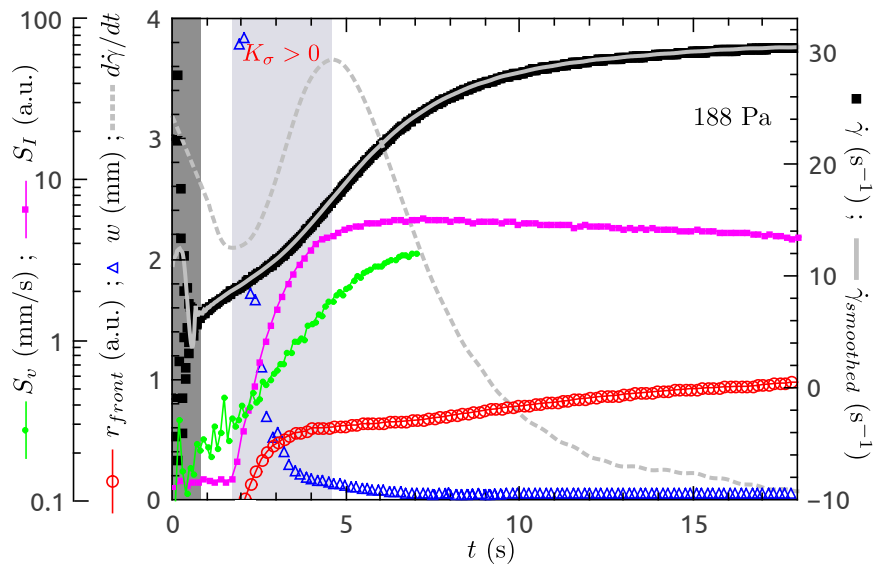

FIG. 7. Comparison of temporal evolution of global and local variables following step stress of values (a) $\sigma=175 \mathrm{~Pa}$, (b) $\sigma=179 \mathrm{~Pa}$, (c) $\sigma=183 \mathrm{~Pa}$, (d) $\sigma=188 \mathrm{~Pa}$ over short time scale. Connected to the right axis are the shear rate response ( $\mathbf{m})$ and its evolution smoothed using a Savitzky-Golay filter (grey line). Connected to the linear left axis are the first derivative of the shear rate $d \dot{\gamma} / d t$ also obtained from the Savitzky-Golay filter (dashed grey line), the position of the front $r_{\text {front }}$ in arbitrary units for clarity of the graph (open red circle) obtained from optical visualisations and the apparent width of the interface $w$ (open blue triangle). Connected to the logarithmic left axis are the square root of the mean of the squared residuals computed from linear fitting of the velocity profiles $\left(S_{v}(t)\right.$ - closed green circle) and the intensity profiles $\left(S_{I}(t)\right.$ - closed pink square). The dark greyed out zone covers the inertio-elastic ringing regime, which is out of interest here. The light greyed out zone delimits the time region where the Moorcroft-Fielding criterion is likely to hold.

locimetry experiments performed by $\mathrm{Hu}$ et al, which are, to the best of our knowledge, the only ones that we can build on for wormlike micelles under step stress protocol (see Fig. 5 in ref. ${ }^{57}$ ). Their data can indeed be revisited under a slightly different angle when considering the actual onset of banding. The velocity profiles recorded with a larger spatial resolution than ours, clearly show some nonlinearity very close to the first inflection point in $\dot{\gamma}(t)$ [i.e., the lower boundary of the unstable regime in $\dot{\gamma}(t)$ according to Eqn (1)] and exhibit significant curvature close to the inner wall.

Finally, let us mention other kinds of protocols we have tested to ascertain the connection between the first inflexion point in $\dot{\gamma}(t)$ and the onset of banding. Different configurations were used both to reduce possible effects of initial creep ringing and to test for the presence of the first inflexion point in $\dot{\gamma}(t)$ and its connexion to the onset of banding.

- The imposition of a constant stress in the plateau region was preceded by an initial fast stress ramp: this protocol suppresses creep ringing. Except for this change at short times, the subsequent evolution is completely similar to the one described for regular step stress in the plateau regime (Fig. 8a).

- A step from rest to $\sigma<\sigma_{p}$ was followed by a step to a stress value belonging to the plateau, the duration of each step being about $60 \mathrm{~s}$. Such a type of protocol is likely to provide a clear distinction between the shear rate response towards a homogeneous state $\left(\sigma<\sigma_{p}\right)$ and the shear-rate response towards a heterogeneous shear-banded state $\left(\sigma \geq \sigma_{p}\right)$. During the first step towards a homogeneous state, constant shear rate time evolution 
(a)

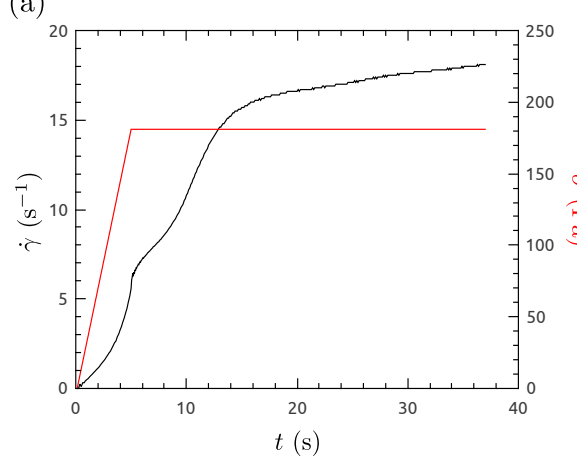

(b)

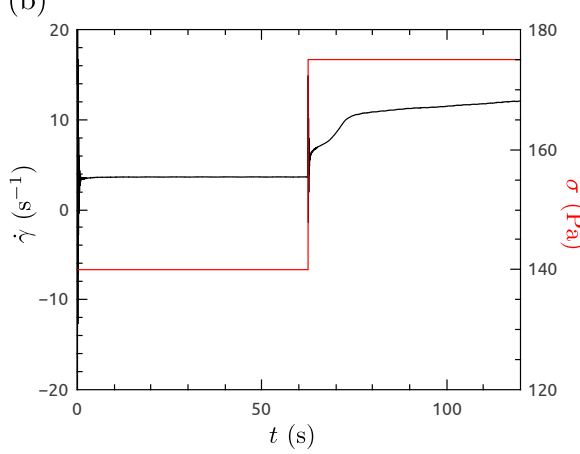

(c)

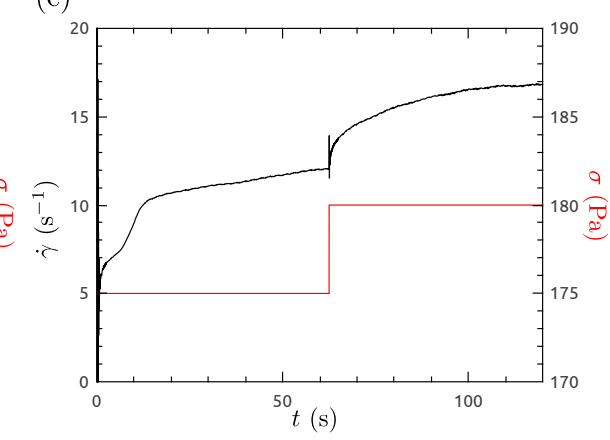

FIG. 8. (a) Time evolution of the shear rate following a stress ramp from 0 to 181 Pa during $5 \mathrm{~s}$ after which the stress is kept constant. The initial ramp allows suppression of creep-ringing. (b) Time evolution of the shear rate following two successive steps stress from $\sigma=0$ to $\sigma=140 \mathrm{~Pa}\left(<\sigma_{p}\right)$ then from $\sigma=140$ to $\sigma=175 \mathrm{~Pa}\left(>\sigma_{p}\right)$. (c) Time evolution of the shear rate following two successive steps stress from $\sigma=0$ to $\sigma=175 \mathrm{~Pa}$ then from $\sigma=175$ to $\sigma=180 \mathrm{~Pa}$. Each step corresponds to shear stress in the shear-banding range.

was observed after creep ringing. After the second step towards a heterogeneous state, the shear rate was found to evolve in the same way as the one described earlier for regular step stress in the plateau regime (Fig. $8 \mathrm{~b}$ ), with the presence of a distinct inflexion point.

- Finally, starting from rest, two successive steps applied during about $60 \mathrm{~s}$ with target stresses inside the plateau were performed. In that case, we expect the system to jump from a banded state to another. Interestingly, if the shear rate response to the first step was found to present an inflexion point and a subsequent regime where Eqn (1) holds, this signature was fully absent in the response to the second step: a monotonic increase with downward curvature was observed before reaching the steady state. Indeed, in this precise case, the banding structure is already established after the first step and during the second step, the system simply adapts the relative proportion of each band (Fig. 8c).

\section{B. The Moorcroft-Fielding shear-banding criterion under step shear rate.}

In contrast to step stress protocol, the response of shear-banding wormlike micelles to shear startup has been widely explored in the literature even over short time scales. If correlation between local and global measurements is not always available and if the time- or space-resolution is not necessarily adapted to capture the onset of banding, some insights are worth mentioning. Note also that experimentally, the onset of banding was often associated with the occurrence of a well-defined kink in the velocity profiles while the process might have started before.
Here we restrict our discussion to applied shear rates in the plateau region (between points A and D in Fig. 1.a), recalling that the ultimate steady state is banded in this entire domain. For applied shear rates along the low shear rate branch, the transient stress response is mostly a mono-exponential increase, as expected for a Maxwellian fluid. As point A in Fig. 1.a is approached, the stress response becomes slightly non-linear ${ }^{47,56}$ and exhibits a small overshoot, the magnitude of which never exceeds $\sigma_{p}$. Note that, in this situation, the relaxation of the small stress overshoot is not associated with transient banding (data not shown).

An example of correlation between time evolution of the shear stress and the velocity profiles can be found in the literature ${ }^{56,57}$. Before the stress overshoot, the velocity measured using particle tracking velocimetry was found to be approximately linear across the gap. The same observation was made by Lettinga et al. using ultrasonic velocimetry on the same system but the shear rate quench was performed from a state along the low shear rate branch ${ }^{72}$. The subsequent behaviour, which extends typically between the stress maximum and the 'foot' of the overshoot (namely the region unstable to banding in Fig. 2.b), was found to depend on the applied shear rate. For the largest explored shear rates, significant wall slip at the inner wall transiently set in before strongly nonlinear velocity profiles were observed probably related to transient development of an (elastic) turbulent burst as observed later on the same system ${ }^{39,40}$ or to shear-induced fracture of the sample. These temporary abnormal velocity profiles were observed to relax towards smooth and slightly curved profiles at a time associated with the 'foot' of the stress overshoot. For lower applied shear rates, no wall slip and no abnormal velocity profiles were reported over the same time interval. After this fast stress relaxation, the velocity profiles were found to progressively curve downward until a kink finally appeared, making the low shear rate 
(a)

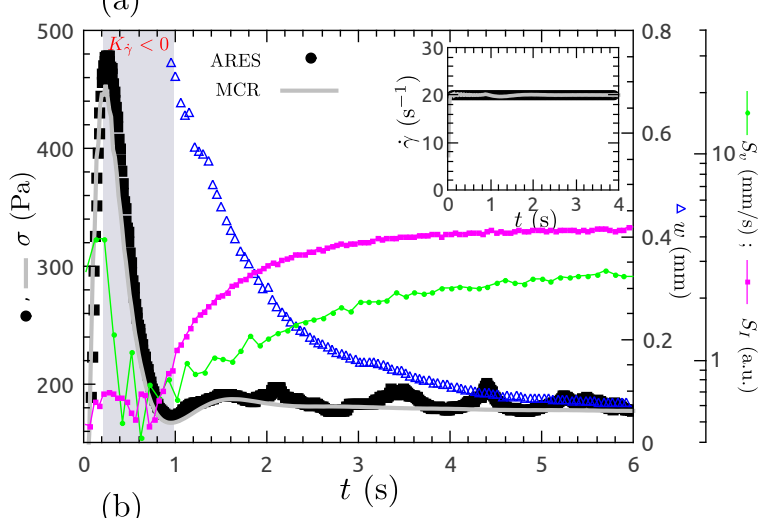

(b)
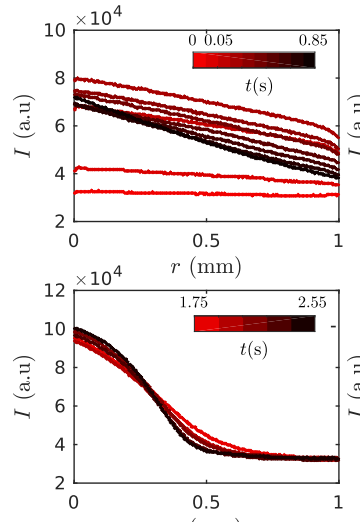

(c)

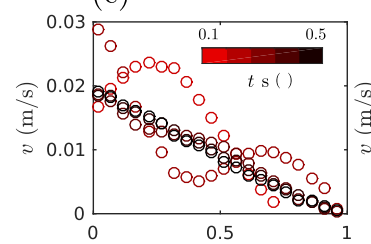

$(\mathrm{mm})$
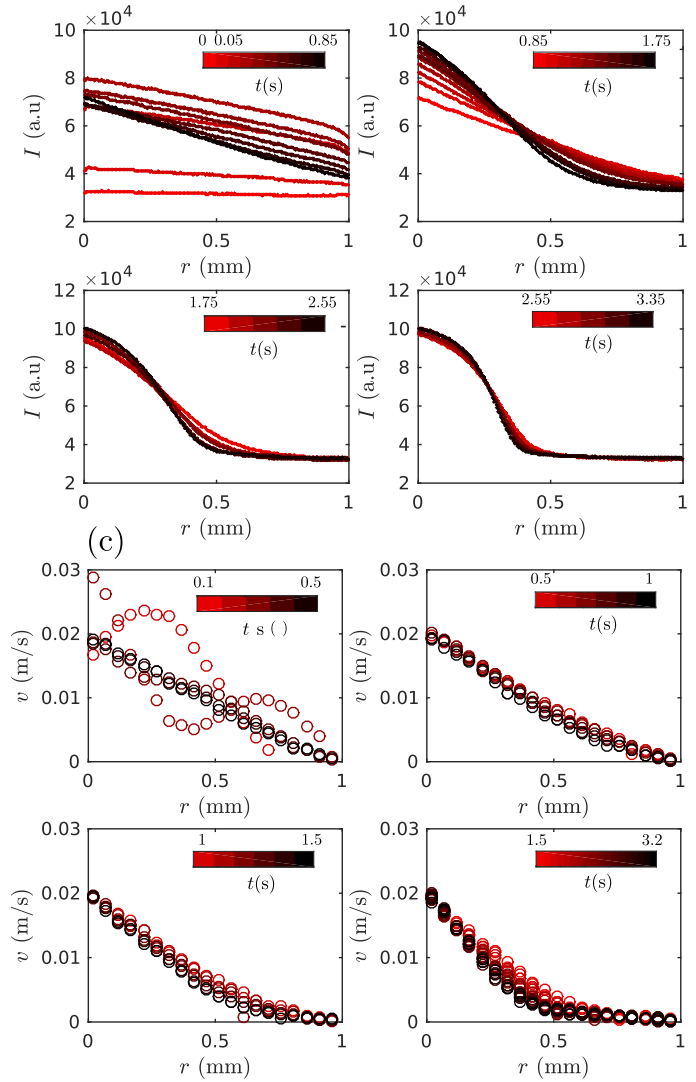

$(\mathrm{mm})$

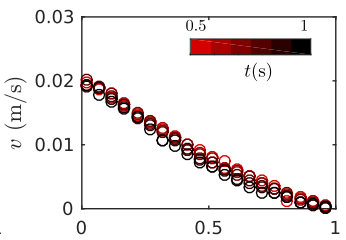

$(\mathrm{mm})$

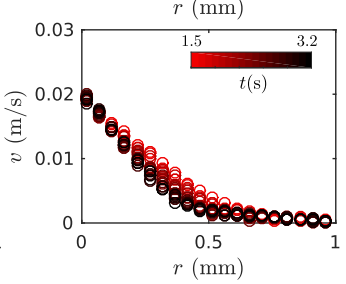

FIG. 9. Comparison between global and local response following step shear rate $\dot{\gamma}=20 \mathrm{~s}^{-1}$. (a) Stress response obtained with an Anton Paar MCR 301 stress-controlled rheometer (grey line) and a TA ARES rheometer operating in strain-controlled mode (closed square). The green circles and pink squares represent $S_{v}(\mathrm{t})$ and $S_{I}(\mathrm{t})$ respectively, while the open triangles represent the apparent width of the interface $w$. Inset: Time evolution of the corresponding imposed shear rate. The light greyed out zone delimits the time region were Eqn (2) holds. (b) Timeresolved intensity profiles recorded simultaneously and covering the first $3.35 \mathrm{~s}$ following shear startup. The actual timeresolution is $0.05 \mathrm{~s}$ but only one in two profiles is displayed to make the reading easier. (c) Time-resolved velocity profiles recorded over the first $3.2 \mathrm{~s}$ with sampling of $0.1 \mathrm{~s}$. In each panel in (b) and (c) the color code goes from red to black as time evolves. band discernible. The following stage was associated with the expansion of the low-shear rate band towards the inner wall together with an increase of the local shear rate in the high-shear rate band. Perge et al. essentially observed the same scenario, with approximately linear velocity profiles until the fast relaxation, after which significant curvature became discernible ${ }^{92}$. Wall slip set in shortly after the stress peak and was found to persist over time (see also ref. ${ }^{39}$ ). Note that delayed formation of the shear-banding structure with respect to the fast overshoot relaxation was also reported for similar wormlike micelles systems in cone-and-plate geometry ${ }^{16,22}$. The velocity profiles were observed to become banded during the sigmoidal relaxation.

Correlation between the stress evolution and the structural organisation of the system has also been reported $40,53,70$. Direct optical visualisations in the $(r, z)$ plane suggested a connection between the stress overshoot and the growth of turbidity of the sample ${ }^{42,70}$. The fast relaxation from the stress peak was associated with homogeneous decrease of the level of turbidity while the subsequent slow relaxation was related to building of a diffuse interface that migrates towards the inner wall and progressively sharpens. This description seems fully compatible with the above observations by $\mathrm{Hu}$ et $a l^{56,57}$. Gurnon et al. reported on the correlation between the transient stress and the evolution of the local microstructure in each band using two-point small angle neutron scattering measurements ${ }^{53}$. The spaceand time-resolution were too limited to accurately determine the onset of the main stages but some insights were found in agreement with the previous findings. Up to the stress peak, the sample was found to be isotropic. Homogeneous alignment across the gap was then observed over a time scale from 5 to $6 \tau$, including the fast stress relaxation and the beginning of the sigmoidal relaxation ${ }^{10}$. Bands of distinct microstructures were found to emerge beyond this time scale.

In practice, the expected region of linear instability of the time-dependent flow [Eqn (2)], namely the fast relaxation of the stress overshoot, often extends over very short time scales for wormlike micelles which makes it difficult to probe with local techniques. In the present study we tried to resolve this region at best, using optical visualisations as the time resolution for the PIV experiments was limited to $0.1 \mathrm{~s}$. Because we used a stress-controlled rheometer, we have checked that the feed-back loop, when operating in strain-controlled mode, did not significantly affect the stress response to shear startup, especially at short times. Comparison between data obtained with a strain-controlled TA ARES rheometer and with our Anton Paar MCR 301 are given in Fig. 9.a. The TC geometry that equipped the ARES was slightly different from ours, with inner radius $R_{i}=16 \mathrm{~mm}$, gap width $d=1 \mathrm{~mm}$, and height $h=33.2 \mathrm{~mm}$, giving a curvature ratio $\Lambda=0.06$ and an aspect ratio $\Gamma=33.2$. Except a slight difference in the 
amplitude of the stress overshoot, the stress responses are consistent. The stress maximum is reached around $t=0.25 \mathrm{~s}$. The fast stress relaxation starting from the peak ends around $t=1 \mathrm{~s}$ and is followed by a damped oscillation $(t \simeq 1.5-2.5 \mathrm{~s})$ and a slower relaxation already described in Part I of this work ${ }^{20}$. The stress overshoot is clearly associated with an increase of the scattered intensity in the whole gap, which also reaches a maximum close to $t=0.25 \mathrm{~s}$ (Fig. 9.b.top-left panel). However, during this process, the level of intensity is not exactly homogeneous in the gap as it was initially suggested by the snapshots in Fig. 4.b: the intensity profiles are approximately linear during the stress growth but the scattered intensity is lower close to the outer wall. As for the velocity profiles, they exhibit complex shapes, with values of the velocity sometimes even larger than the velocity imposed at the moving wall: such shapes at very early times have not been reported earlier. They might be related to temporary 3D flow and/or elastic recoil ${ }^{84,106 .}$ Since this peculiar behavior arises over the time interval preceding the stress maximum, it might potentially mask a very fine comparison with Moorcroft-Fielding criterion. However, just after the stress peak $(t \simeq 0.3 \mathrm{~s})$, the velocity profiles become approximately linear, in agreement with observations by Hu et al. ${ }^{56,57}$ or Perge et al. ${ }^{92}$. This is observed in fig. 9.c.top-left panel and shown by the abrupt drop of $S_{v}$ in fig. 9.a. The first signs of departure from the linear profile appear at the end of the fast stress relaxation for $t \simeq 1 \mathrm{~s}$ (Fig. 9.c.top-right panel) where $S_{v}$ starts increasing out of the noise. Curvature also starts becoming detectable in the intensity profile from $t \simeq 0.95 \mathrm{~s}$, especially close to the fixed wall (see Fig. 9.b.top-right panel). Emergence of structural heterogeneity at that time is confirmed by the evolution of $S_{I}$ as a function of time (Fig. 9.a). This corresponds to the formation of a diffuse front that progressively sharpens as illustrated by the evolution of the apparent width $w$ of the interface (Fig. 9.a) and moves towards the inner wall (see also Fig. 9.b.bottom panels).

These observations confirm the body of data accumulated by other groups: the onset of banding in shear startup protocol does not start at or slightly before the stress overshoot as predicted by Eqn (2). Shear banding is more likely triggered very slightly before the end of the fast stress overshoot relaxation. The sharpening of the interface between bands and the emergence of an effective kink in the velocity profiles occur during the slow relaxation towards steady state.

In the next section, we examine whether the situation observed for other complex fluids under the same type of time-dependent flow protocols presents similarities with the case of shear-banding wormlike micelles.

\section{Comparison with other complex fluids.}

Polymer solutions and polyelectrolytes systems such as DNA solutions under time-dependent flow protocols have been probed using combination of global rheology and particle tracking velocimetry $14,15,17-19,55,58,73,74,97$. Note that only refs. ${ }^{14,55,58}$ dealt with TC flow. Here, we put aside the controversy about the effective existence of shear banding in polymer solutions as an intrinsic property of the system ${ }^{73,74,110}$. Overall, from the available experimental data, it is not simple to extract robust features as in the case of micellar systems, which makes a direct quantitative comparison very difficult. Therefore we limit ourselves to recalling some of the main observations. After a step stress, the shear rate response as a function of time of DNA solutions was found to roughly follow the sketch depicted in Fig. 2. ${ }^{17,18}$, with an initial slow creep regime followed by a rapid increase of the strain rate. In polybutadiene solutions, the initial creep regime was replaced by an induction period where the shear rate was essentially constant or slightly increasing $^{55}$. The creep regime (or the induction period) seemed dominated by wall slip while the subsequent raise in $\dot{\gamma}$ was characterized by nonlinear velocity profiles which may become banded at long times. After shear startup, the expected shear stress overshoot was observed. Before the stress peak the velocity profiles were found to be linear. During the overshoot relaxation, just after the stress peak, plug-like flow with wall slip at both walls and sometimes elastic recoil were observed. Curved and eventually banded profiles were rather found to develop close to the 'foot' of the stress overshoot. In all the experiments cited above, the accurate correlation between the fine variations of the rheological global signals and the temporal evolution of the local flow field is not possible because the time-resolution of the velocimetry data appears inadequate or because velocimetry data are simply lacking in the time window of interest, which precludes the accurate test of the Moorcroft-Fielding criteria.

In soft glassy materials, the response of different classes of yield stress fluids (simple or aging and thixotropic YSF) under such time-dependent flow protocols has been investigated in depth by combining global rheology measurements and time- and space-resolved velocimetry $31,32,34,49,50,82$.

Fig. 10.a displays the typical shear rate response to step stress of carbopol microgel, a soft repulsive glass that behaves as a simple YSF. The steady-state flow curve of this kind of fluids follows the Herschel-Bulkley $\operatorname{model}\left(\sigma=\sigma_{y}+\eta \dot{\gamma}^{n}\right.$, with $\sigma_{y}$ the yield stress and $n$ the shear-thinning exponent) and shear banding is only transient, the steady-state flow behavior being characterized by a uniform shear flow. When represented in log-log scale, the shear rate was found to adopt a S-shaped curve, compatible with the sketch in Fig. 2.a, with an initial power law decay followed by a strong increase of $\dot{\gamma}(t)$ before a steady state was reached (see left inset in Fig. 10.a). A similar evolution was also observed for weakly attractive thixotropic colloidal (carbon black) gels ${ }^{50}$. At first sight, despite very different microstructures that lead to different early dynamics (inertio-elastic ringing for mi- 
(a)

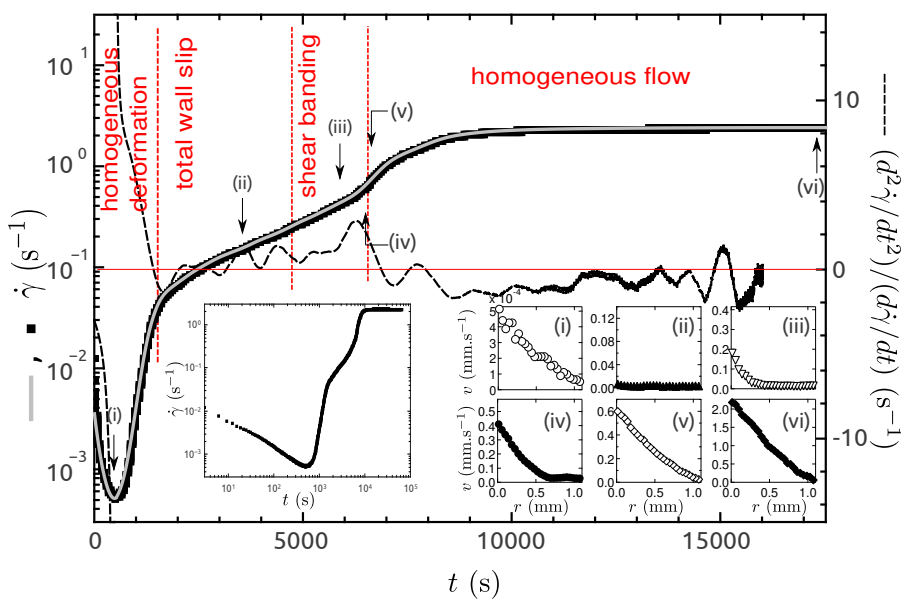

(b)

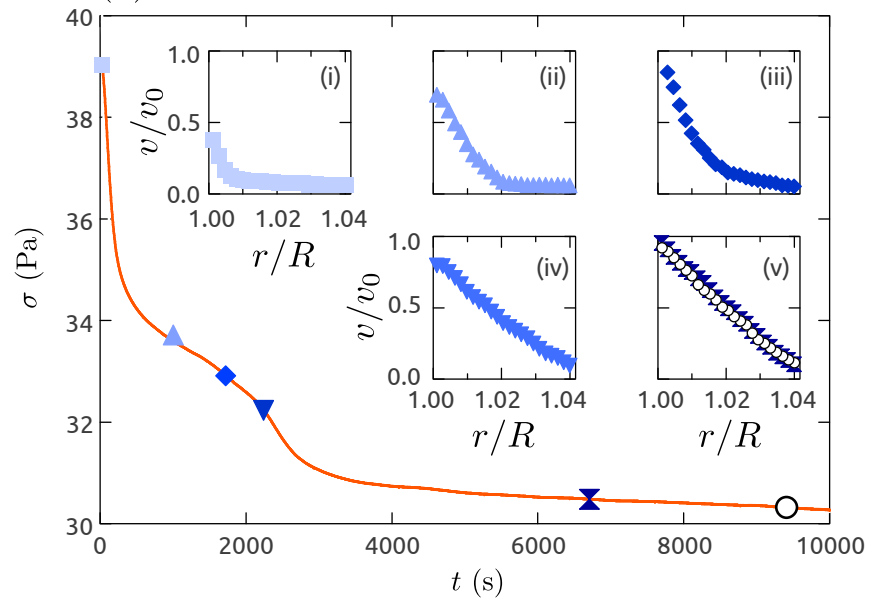

FIG. 10. Time-dependent flow of a carbopol microgel. (a) Shear rate $\dot{\gamma}$ vs time $t$ for a shear stress $\sigma=37$ Pa applied at $t=0$ under rough boundary conditions ( $\boldsymbol{\square})$. The grey line corresponds to $\dot{\gamma}(t)$ smoothed using Savitzky-Golay filter. This filter is also used to provide the ratio between the second and the first derivatives (dashed line). Insets: (Left) Log-log plot of $\dot{\gamma}(t)$. (Right) Velocity profiles $v(r)$, where $r$ is the distance to the rotor, at different times labelled by the different symbols in the $\dot{\gamma}(t)$ curve. On each profile, the upper value of the velocity scale is set to the current rotor velocity $v_{0}$. Adapted with permission from Divoux et al. ${ }^{32}$, copyright by the Royal Society of Chemistry. (b) Shear stress $\sigma$ vs time for an imposed shear rate $\dot{\gamma}=0.7 \mathrm{~s}^{-1}$. Insets: Normalized velocity profiles $v / v_{0}$ at different times indicated in the main panel using the same symbols. Reprinted with permission from Divoux et al. ${ }^{33}$, copyright by Annual Reviews.

celles versus creep deformation for YSF) and very different time scales, the overall shape of the shear rate time evolution following step stress presents some similarities. Beyond the early dynamics, in the two cases, $\dot{\gamma}$ is an increasing function of time and exhibits similar successive changes of curvature. However, the corresponding behaviour at local scale is somewhat different.

Using simultaneous ultrasonic velocimetry measurements, different stages were identified along the shear rate response: the microgel first experiences homogeneous elastic deformation. The velocity profiles are approximately linear and involve extremely small velocities [Fig. 10.a.(i)]. The subsequent stage, whatever the type of boundary conditions, smooth or rough, corresponds to total wall slip [Fig. 10.a.(ii)]. Nucleation and growth of a shear band at the rotor is then observed [Fig. 10.a.(iii)-(iv)]. The final stage corresponds to full fluidisation of the sample, after which the velocity profiles remain linear while wall slip vanishes [Fig. 10.a.(v)(vi)]. In Fig. 10, we have added the ratio between the second and the first derivatives of the shear rate versus time obtained using Savitzky-Golay filter in order to visualise more easily the domains where the MoorcroftFielding criterion [Eqn (1)] is likely to hold. As schematized in Fig. 2.a, Eqn (1) holds at early times when the first derivative becomes positive around $t \simeq 500 \mathrm{~s}$, the local curvature being positive as well but this regime is associated with homogeneous deformation characterized by a linear velocity profile and not with shear banding. Beyond the minimum in $\dot{\gamma}(t)$, the first derivative $d \dot{\gamma} / d t$ is always positive while several successive changes of curvature are visible. Disregarding the 'fast' (in comparison to the time scales of the different stages) local changes of curvature related to inherent noise of the experiment, the shear-banding criterion starts holding around $t \simeq 3000-4000 \mathrm{~s}$, inside the total wall slip regime and it is also valid in the following shear banding regime. Finally, the subsequent change of curvature, as $d^{2} \dot{\gamma} / d t^{2}$ becomes negative ( $t \lesssim 7000 \mathrm{~s}$ ) seems related to the transition towards the homogeneous flow state.

In contrast to wormlike micelles, the first inflexion point where the local curvature goes from negative to positive, does not exactly coincide with the onset of banding. In addition, the regime of total wall slip that might generally go hand in hand with shear banding for colloidal gels as well as for repulsives glasses, is absent in wormike micelles. Interestingly, for wormlike micelles, wall slip emerges when the shear-induced structure nucleates from the inner wall, as illustrated in Fig. 11, which compares the velocity at the inner wall computed from the global shear rate response and the local velocity at the wall measured using PIV. Departure between the two seems to happen slightly after the onset of banding, confirming that wall slip is intimately connected to the properties of the shear-induced structures in the highshear rate band. In contrast, for YSF wall slip is likely to act as a precursor to bulk shear banding.

In startup flow configuration, the shear stress response of simple yield stress fluids was found to exhibit a stress overshoot $^{32}$ as illustrated in Fig. 10.b for carbopol microgels. Stress overshoots were also observed in aged thixotropic materials such as laponite suspensions ${ }^{49,82}$, 


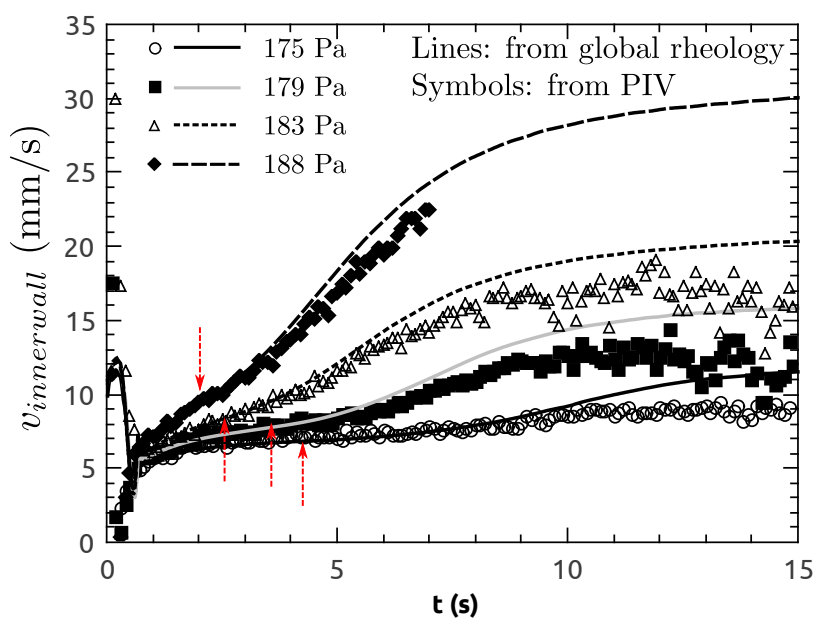

FIG. 11. Velocity at the inner wall as a function of the time for various applied steps stress in the banding regime of the wormlike micelles system. The lines correspond to the velocity expected at the inner wall from the global shear rate measurements. The symbols provide the local velocity at the inner wall obtained from PIV measurements. The red arrows indicate the onset of banding as determined from Fig. 7.

waxy crude oils ${ }^{30}$, colloidal suspensions ${ }^{28}$ and colloidal gels $^{29,67}$. In both cases, transient shear banding was observed shortly after the stress overshoot and during the stress decay towards steady-state [see Fig. 10.b.(i)(ii)-(iii)], consistently with predictions by Moorcroft and Fielding $44,85,86$. Full fluidisation of the samples was finally observed after this long-lived shear-banding regime [see Fig. 10.b.(iv)-(v)]. Wall slip was again observed throughout the (transient) shear-banding regime whatever the boundary conditions while it was found to be insignificant in the ultimate homogeneous steady state at least under rough boundary conditions.

Again, the global response is similar to the case of wormlike micelles even if the time scales that are involved are very different. For YSF, the onset of banding is clearly connected to the relaxation of the stress overshoot in agreement with Eqn (2) while it is delayed for wormlike micelles. In the two cases, wall slip is present. In wormlike micelles slip sets in while the shear-induced structures are nucleated, that is at the stress ovsershoot, and seems to precede the onset of banding.

\section{CONCLUSIONS}

In this article, we have studied the global and local responses of a well-known shear-banding micellar system to common time-dependent flow protocols such as step stress and shear startup. The main objective was to test the validity of shear-banding criteria established by Moorcroft and Fielding within a general theoretical framework encompassing both complex fluids and soft solids ${ }^{44,85-87}$. These criteria, which are associated with characteristic mechanical signatures, suggest the presence of banding (or not) in the underlying flow field solely on the basis of the time evolution of the measured bulk rheological signals, even for systems that do not exhibit shear banding as ultimate steady-state. Beyond the case of shear-banding wormlike micelles, we have examined data available for simple YSF, for which shear banding is only transient. Direct comparison between the two types of systems shows that, if the overall time evolution of the global rheological signals present some strong similarities, the correlation with the response at local scale differs and a strict application of the Moorcroft-Fielding criterion can be misleading.

The whole picture is summarized in figure 12. For step stress protocol, the short-time response is very different for both types of systems: $\dot{\gamma}(t)$ is dominated by inertio-elastic ringing for wormlike micelles while creep deformation characterized by a power law decay is observed for simple YSF. The flow field is homogeneous just after creep ringing in wormlike micelles and during creep deformation in YSF. Beyond this early stage, the subsequent evolution of $\dot{\gamma}(t)$ for both fluids is a monotonic increase towards steady state. For simple YSF, $\dot{\gamma}(t)$ simultaneously curves and slopes upward at the transition from creep to yielding. According to Eqn (1), the fluid is predicted to be unstable to the formation of shear bands. However, the experiments show that homogeneous deformation persists in this time interval. Beyond that, $\dot{\gamma}(t)$ keeps sloping upward and exhibits successive changes of curvature very similar to those observed for wormlike micelles. For both fluids, a time window can be identified where $\dot{\gamma}(t)$ exhibits positive curvature so that Eqn (1) holds. For wormlike micelles, we have shown that the onset of banding arises inside this window of instability. Clear structural heterogeneity with a diffuse front between bands emerges while the velocity profiles become nonlinear and slip at the inner wall occurs. The stiffening process of the front in both the structural and velocity profiles starts within the instability window while the formation of a distinct kink in the shear-band profile occurs after the time window of instability. For simple YSF, total wall slip precedes shear banding in the unstable region. Note that for the two types of complex systems, the observed mechanical signature does not exactly coincide with the one expected from the models tested numerically. It seems more likely related to a change of curvature from negative to positive rather than a change of slope.

Under shear startup protocol, the stress response for the two systems is dominated by an overshoot. Shear banding is observed together with wall slip just after the stress peak in YSF, in full agreement with the MoorcroftFielding criterion. For wormlike micelles, the situation is different. Hints of structural and flow field heterogeneity are evidenced very slightly before the end of the fast stress overshoot relaxation, the onset of banding being delayed with respect to the expected window of instability. Nevertheless, the stress overshoot clearly acts as 

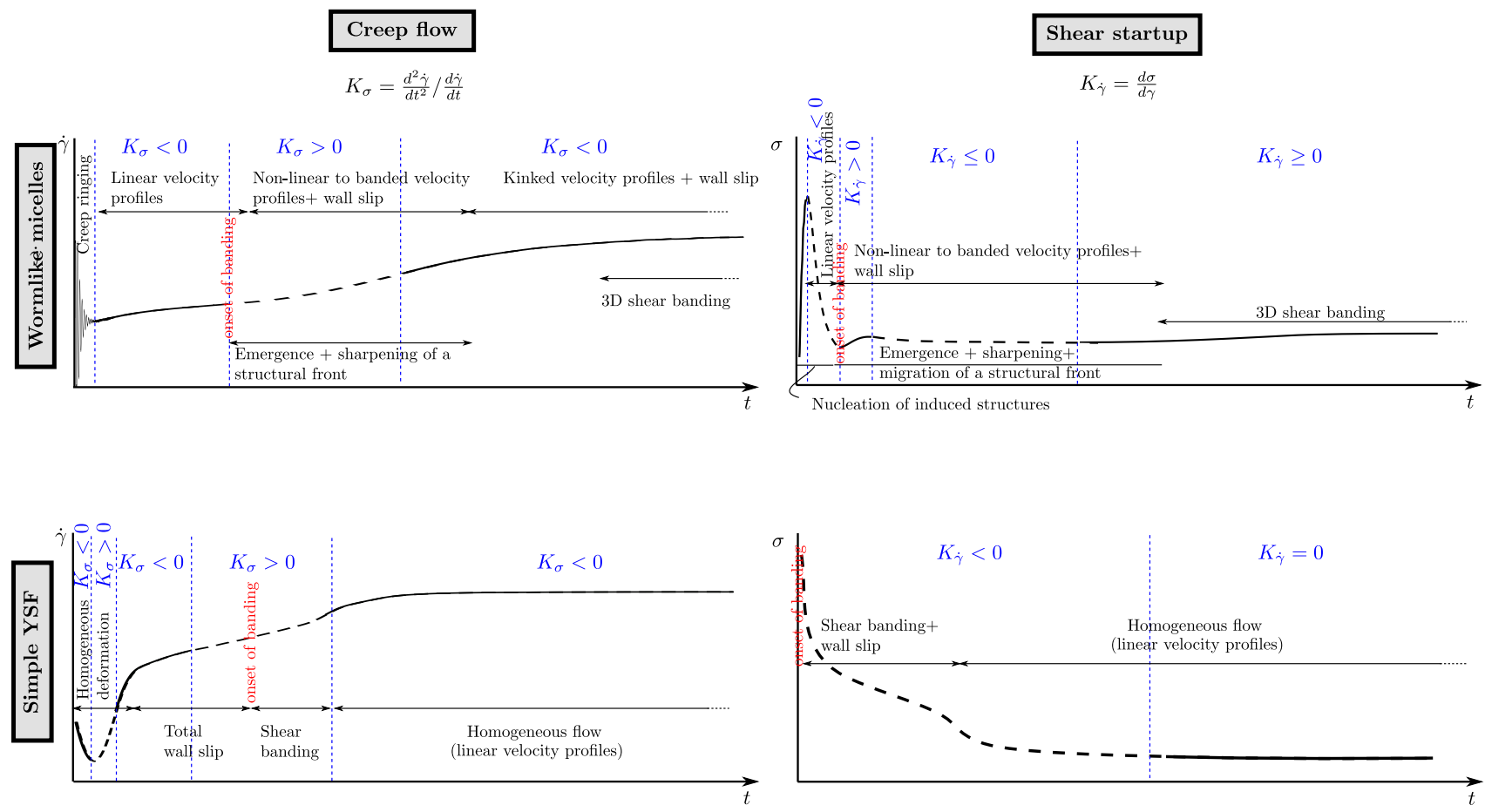

FIG. 12. Schematic view of the mechanical response of wormlike micelles (top) and simple YSF (bottom) under step shear stress (left) and shear startup (right). This scheme echoes figure 2. In each case, we specified the ranges where the Moorcroft-Fielding criteria are valid and the behaviours observed at local scale. In these sketches, the scale of the time axis is linear.

a precursor to banding in wormlike micellar systems since the shear-induced structures are nucleated during this stage.

From these sets of data, we suggest that the criteria based on specific variations of the bulk rheological signals appear as necessary but not sufficient conditions for the onset of banding. They constitute indicators in favour of banding but should not be used without complementary local measurements to claim the existence of banding or not, either transient or steady. Experimentally, whatever the microscopic details of the structure of the systems, shear-banding and wall slip appear strongly intertwined. A milestone would be the incorporation of wall slip as a key ingredient in the models predicting shear banding in time-dependent and steady-state flows. Effects of flow-concentration coupling could also be considered in the time-dependent evolution of the global mechanical variables, especially for wormlike micelles where the emergence of turbidity may be indicative of such an effect. Finally, the Moorcroft-Fielding criteria have been established for planar Couette flow and the possible impact of the curvature inherent to the TC flow remains to be clarified. Possible effects of fluid inertia during flow startup should also be examined ${ }^{65}$.

\section{ACKNOWLEDGMENTS}

This work benefited from meetings within the French working group GDR CNRS 2019 'Solliciter La Matière Molle' (SLAMM). The authors thank Thibaut Divoux, Sébastien Manneville and Suzanne Fielding for the critical reading of the manuscript.

${ }^{1}$ Adams, J. and Olmsted, P., "Nonmonotonic models are not necessary to obtain shear banding phenomena in entangled polymer solutions," Physical review letters 102, 067801 (2009).

${ }^{2}$ Adams, J. M., Fielding, S. M., and Olmsted, P. D., “Transient shear banding in entangled polymers: A study using the Rolie-Poly model," J. Rheol. 55, 1007-1032 (2011).

${ }^{3}$ Baravian, C., Benbelkacem, G., and Caton, F., "Using instrumental inertia in controlled stress rheometry," Rheol. Acta 46, 577-581 (2007).

4Bautista, F., Pérez-López, J., García, J., Puig, J., and Manero, O., "Stability analysis of shear banding flow with the bmp model," Journal of Non-Newtonian Fluid Mechanics 144, 160-169 (2007).

${ }^{5}$ Bautista, F., Soltero, J. F. A., Macías, E. R., Puig, J. E., and Manero, O., "Irreversible thermodynamics approach and modeling of shearbanding flow of wormlike micelles," The Journal of Physical Chemistry B 106, 13018-13026 (2002), https://doi.org/10.1021/jp0206370.

${ }^{6}$ Bécu, L., Manneville, S., and Colin, A., "Yielding and flow in adhesive and nonadhesive concentrated emulsions," Phys. Rev. Lett. 96, 138302 (2006)

${ }^{7}$ Benzi, R., Divoux, T., Barentin, C., Manneville, S., Sbragaglia, M. and Toschi, F., "Unified theoretical and experimental view on transient shear banding," Phys. Rev. Lett. 123, 248001 (2019).

${ }^{8}$ Benzi, R., Sbragaglia, M., Bernaschi, M., Succi, S., and Toschi, F., "Cooperativity flows and shear-bandings: a statistical field theory approach," Soft Matter 12, 514-530 (2016).

${ }^{9}$ Berret, J., "Rheology of wormlike micelles: Equilibrium properties 
and shear banding transitions," in Molecular Gels, edited by R. G. Weiss and P. Terech (Springer Netherlands, 2006) pp. 667-720.

${ }^{10}$ Berret, J.-F., "Transient rheology of wormlike micelles," Langmuir 13, 2227 (1997).

${ }^{11}$ Besseling, R., Ballesta, L. I. P., Petekidis, G., Cates, M., and Poon, W., "Shear banding and flow-concentration coupling in colloidal glasses," Phys. Rev. Lett. 105, 268301 (2010).

${ }^{12}$ Bocquet, L., Colin, A., and Ajdari, A., "A kinetic theory of plastic flow in soft glassy materials," Phys. Rev. Lett. 103, 036001 (2009)

${ }^{13}$ Bonn, D., Denn, M. M., Berthier, L., Divoux, T., and Manneville, S., "Yield stress materials in soft condensed matter," Rev. Mod. Phys. 89, 035005 (2017)

${ }^{14}$ Boukany, P. E., Hu, Y. T., and Wang, S.-Q., “Observations of wall slip and shear banding in an entangled dna solution," Macromolecules 41, 2644-2650 (2008), https://doi.org/10.1021/ma702332n.

${ }^{15}$ Boukany, P. E. and Wang, S.-Q.," "A correlation between velocity profile and molecular weight distribution in sheared entangled polymer solutions," J. Rheol. 51, 217-233 (2007).

${ }^{16}$ Boukany, P. E. and Wang, S.-Q., "Use of particle-tracking velocimetry and flow birefringence to study nonlinear flow behavior of entangled wormlike micellar solution: from wall slip, bulk disentanglement to chain scission," Macromolecules 41, 1455-1464 (2008), https://doi.org/10.1021/ma702527s.

${ }^{17}$ Boukany, P. E. and Wang, S.-Q., "Exploring the transition from wall slip to bulk shearing banding in well-entangled DNA solutions," Soft Matter 5, 780-789 (2009).

${ }^{18}$ Boukany, P. E. and Wang, S.-Q., "Shear banding or not in entangled DNA solutions depending on the level of entanglement," J. Rheol. 53, 73-83 (2009).

${ }^{19}$ Boukany, P. E. and Wang, S.-Q., "Shear Banding or Not in Entangled DNA Solutions," Macromolecules 43, 6950-6952 (2010).

${ }^{20}$ Briole, A., Casanellas, L., Fardin, M.-A., Py, C., Cardoso, O., Browaeys, J., and Lerouge, S., "Shear-banding wormlike micelles under time-dependent flows: flow dynamics," Soft Matter , submitted (2021).

${ }^{21}$ Britton, M. M. and Callaghan, P. T., "Two-phase shear band structures at uniform stress," Phys. Rev. Lett. 78, 4930-4933 (1997).

${ }^{22}$ Casanellas, L., Dimitriou, C., Ober, T., and McKinley, G., "Spatiotemporal dynamics of multiple shear-banding events for viscoelastic micellar fluids in cone-plate shearing flows," J. non-Newt. Fluid Mech. , 234-247 (2015).

${ }^{23}$ Cates, M. E., "Nonlinear viscoelasticity of wormlike micelles (and other reversibly breakable polymers)," J. Phys. Chem. 94, 371-375 (1990).

${ }^{24}$ Cates, M. E. and Fielding, S. M., "Rheology of giant micelles," Advances in Physics 55, 799-879 (2006).

${ }^{25}$ Coussot, P., Nguyen, Q. D., Huynh, H. T., and Bonn, D., "Avalanche behavior in yield stress fluids," Phys. Rev. Lett. 88, 175501 (2002).

${ }^{26}$ Cromer, M., Fredrickson, G. H., and Leal, L. G., "A study of shear banding in polymer solutions," Physics of Fluids 26, 063101 (2014), https://doi.org/10.1063/1.4878842.

${ }^{27}$ Cromer, M., Villet, M. C., Fredrickson, G. H., and Leal, L. G., "Shear banding in polymer solutions," Physics of Fluids 25, 051703 (2013), https://doi.org/10.1063/1.4805089.

${ }^{28}$ Derec, C., Ducouret, G., Ajdari, A., and Lequeux, F., "Aging and nonlinear rheology in suspensions of polyethylene oxide-protected silica particles," Phys. Rev. E 67, 061403 (2003).

${ }^{29}$ Derec, C., Senis, D., Talini, L., and Allain, C., "Rapid settling of a colloidal gel," Phys. Rev. E 67, 062401 (2003).

${ }^{30}$ Dimitriou, C. J. and McKinley, G. H., "A comprehensive constitutive law for waxy crude oil: a thixotropic yield stress fluid," Soft Matter - (2014).

${ }^{31}$ Divoux, T., Barentin, C., and Manneville, S., "From transient fluidization processes to herschel-bulkley behavior in simple yield stress fluids," Soft Matter 7, 8409-8418 (2011)

${ }^{32}$ Divoux, T., Barentin, C., and Manneville, S., "Stress overshoot in a simple yield stress fluid: an extensive study combining rheology and velocimetry," Soft Matter 7, 9335-9349 (2011).

${ }^{33}$ Divoux, T., Fardin, M. A., Manneville, S., and Lerouge, S., "Shear banding of complex fluids," Annual Review of Fluid Mechanics 48,
81-103 (2016), https://doi.org/10.1146/annurev-fluid-122414-034416.

${ }^{34}$ Divoux, T. Tamarii, D., Barentin, C., and Manneville, S., "Transient shear banding in a simple yield stress fluid," Phys. Rev. Lett. 104, 208301 (2010).

${ }^{35}$ Divoux, T., Tamarii, D., Barentin, C., Teitel, S., and Manneville, S., "Yielding dynamics of a herschel-bulkley fluid: a critical-like fluidization behaviour," Soft Matter 8, 4151-4164 (2012).

${ }^{36}$ Doi, M. and Edwards, S., The theory of polymer dynamics, Vol. 73 (Oxford University Press, USA, 1988).

${ }^{37}$ Ewoldt, R. H., Johnston, M. T., and Caretta, L. M., “Experimental challenges of shear rheology: How to avoid bad data," in Complex Fluids in Biological Systems: Experiment, Theory, and Computation edited by S. E. Spagnolie (Springer New York, New York, NY, 2015) pp. 207-241.

${ }^{38}$ Ewoldt, R. H., Winegard, T. M., and Fudge, D. S., "Non-linear viscoelasticity of hagfish slime," International Journal of Non-Linear Mechanics 46, 627-636 (2011), special issue on non-linear mechanics of biological structures.

${ }^{39}$ Fardin, M., Divoux, T., Guedeau-Boudeville, M., Buchet-Maulien, I. Browaeys, J., McKinley, G., Manneville, S., and Lerouge, S., "Shearbanding in surfactant wormlike micelles: elastic instabilities and wall slip," Soft Matter 8, 2535-2553 (2012)

${ }^{40}$ Fardin, M.-A., Casanellas, L., Saint-Michel, B., Manneville, S., and Lerouge, S., "Shear-banding in wormlike micelles: Beware of elastic instabilities," J. Rheol. 60, 917-926 (2016), https://doi.org/10.1122/1.4960333.

${ }^{41}$ Fardin, M. A., Ober, T. J., Grenard, V., Divoux, T., Manneville, S. McKinley, G. H., and Lerouge, S., "Interplay between elastic instabilities and shear-banding: three categories of Taylor-Couette flows and beyond," Soft Matter 8, 10072-10089 (2012).

${ }^{42}$ Fardin, M.-A., Radulescu, O., Morozov, A., Cardoso, O., Browaeys, J., and Lerouge, S., "Stress diffusion in shear banding wormlike micelles," J. Rheol. 59, 1335-1362 (2015) https://doi.org/10.1122/1.4930858.

${ }^{43}$ Fielding, S. M., "Shear banding in soft glassy materials," Reports on Progress in Physics 77, 102601 (2014).

${ }^{44}$ Fielding, S. M., "Triggers and signatures of shear banding in steady and time-dependent flows," J. Rheol. 60, 821-834 (2016), https://doi.org/10.1122/1.4961480.

${ }^{45}$ Fielding, S. M., Cates, M. E., and Sollich, P., "Shear banding, aging and noise dynamics in soft glassy materials," Soft Matter 5, 2378 2382 (2009).

${ }^{46}$ Fielding, S. M. and Olmsted, P. D., "Flow phase diagrams for concentration-coupled shear banding," Eur. Phys. J. E 11, 65-83 (2003).

${ }^{47}$ Fischer, P. and Rehage, H., "Rheological master curves of viscoelastic surfactant solutions by varying the solvent viscosity and temperature," Langmuir 13, 7012-7020 (1997).

${ }^{48}$ Geri, M. Venkatesan, R., Sambath, K., and McKinley, G. H., "Thermokinematic memory and the thixotropic elastoviscoplasticity of waxy crude oils," Journal of Rheology 61, 427-454 (2017), https://doi.org/10.1122/1.4978259.

${ }^{49}$ Gibaud, T., Barentin, C., and Manneville, S., "Influence of boundary conditions on yielding in a soft glassy material," Phys. Rev. Lett. 101, 258302 (2008)

${ }^{50}$ Gibaud, T., Frelat, D., and Manneville, S., "Heterogeneous yielding dynamics in a colloidal gel," Soft Matter 6, 3482-3488 (2010).

${ }^{51}$ Giesekus, H., "A simple constitutive equation for polymer fluids based on the concept of deformation-dependent tensorial mobility," JNNFM 11, 69 - 109 (1982)

${ }^{52}$ Grenard, V., Divoux, T., Taberlet, N., and Manneville, S. "Timescales in creep and yielding of attractive gels," Soft Matter 10, 1555-1571 (2014)

${ }^{53}$ Gurnon, A. K., Lopez-Barron, C., Wasbrough, M. J., Porcar, L., and Wagner, N. J., "Spatially resolved concentration and segmental flow alignment in a shear-banding solution of polymer-like micelles," ACS Macro Letters 3, 276-280 (2014) http://pubs.acs.org/doi/pdf/10.1021/mz5000152.

${ }^{54}$ Hinkle, A. R. and Falk, M. L., "A small-gap effective-temperature model of transient shear band formation during flow," J. Rheol. 60 
873-882 (2016), https://doi.org/10.1122/1.4960514.

${ }^{55} \mathrm{Hu}, \mathrm{Y}$. T., "Steady-state shear banding in entangled polymers?" J. Rheol. 54, 1307-1323 (2010)

${ }^{56} \mathrm{Hu}$, Y. T. and Lips, A., "Kinetics and mechanism of shear banding in an entangled micellar solution," J. Rheol. 49, 1001-1027 (2005).

${ }^{57} \mathrm{Hu}, \mathrm{Y}$. T., Palla, C., and Lips, A., "Comparison between shear banding and shear thinning in entangled micellar solutions," J. Rheol. 52, 379-400 (2008).

${ }^{58} \mathrm{Hu}, \mathrm{Y}$. T., Wilen, L., Philips, A., and Lips, A., "Is the constitutive relation for entangled polymers monotonic?" J. Rheol. 51, 275-295 (2007).

${ }^{59}$ Illa, X., Puisto, A., Lehtinen, A., Mohtaschemi, M., and Alava, M., "Transient shear banding in time-dependent fluids," Phys. Rev. E 87, 022307 (2013)

${ }^{60}$ Jain, A., Singh, R., Kushwaha, L., Shankar, V., and Joshi, Y. M., "Role of inertia and thixotropy in start-up flows of aging soft materials: Transient dynamics and shear banding in a ratecontrolled flow field," Journal of Rheology 62, 1001-1016 (2018), https://doi.org/10.1122/1.5023305.

${ }^{61}$ Jaradat, S., Harvey, M., and Waigh, T. A., "Shear-banding in polyacrylamide solutions revealed via optical coherence tomography velocimetry," Soft Matter 8, 11677-11686 (2012).

${ }^{62}$ Jin, H., Kang, K., Ahn, K. H., and Dhont, J. K. G., "Flow instability due to coupling of shear-gradients with concentration: non-uniform flow of (hard-sphere) glasses," Soft Matter 10, 9470-9485 (2014).

${ }^{63}$ Johnson, M. and Segalman, D., "A model for viscoelastic fluid behavior which allows non-affine deformation," JNNFM 2, 255 - 270 (1977).

${ }^{64}$ Kapur, J., Sahoo, P., and Wong, A., "A new method for gray-level picture thresholding using the entropy of the histogram," Computer Vision, Graphics, and Image Processing 29, 273 - 285 (1985)

${ }^{65}$ Korhonen, M., Mohtaschemi, M., Puisto, A., Illa, X., and Alava, M. J., "Start-up inertia as an origin for heterogeneous flow," Phys. Rev. E 95, 022608 (2017)

${ }^{66}$ Kunita, I., Sato, K., Tanaka, Y., Takikawa, Y., Orihara, H., and Nakagaki, T., "Shear banding in an f-actin solution," Phys. Rev. Lett. 109, 248303 (2012)

${ }^{67}$ Kurokawa, A., Vidal, V., Kurita, K., Divoux, T., and Manneville, S., "Avalanche-like fluidization of a non-brownian particle gel," Soft Matter 11, 9026-9037 (2015).

${ }^{68}$ Lerouge, S., Argentina, M., and Decruppe, J.-P., "Interface instability in shear-banding flow," Phys. Rev. Lett. 96, 088301 (2006).

${ }^{69}$ Lerouge, S. and Berret, J.-F., "Shear-induced transitions and instabilities in surfactant wormlike micelles," Adv Polym Sci 230, 1-71 (2010).

${ }^{70}$ Lerouge, S., Fardin, M. A., Argentina, M., Gregoire, G., and Cardoso, O., "Interface dynamics in shear-banding flow of giant micelles," Soft Matter 4, 1808-1819 (2008).

${ }^{71}$ Lerouge, S. and Olmsted, P. D., "Non-local effects in shear banding of polymeric flows," Frontiers in Physics 7, 246 (2020).

${ }^{72}$ Lettinga, M. P. and Manneville, S., "Competition between shear banding and wall slip in wormlike micelles," Phys. Rev. Lett. 103, 248302 (2009)

${ }^{73} \mathrm{Li}$, Y., Hu, M., McKenna, G. B., Dimitriou, C. J., McKinley, G. H., Mick, R. M., Venerus, D. C., and Archer, L. A., "Flow field visualization of entangled polybutadiene solutions under nonlinear viscoelastic flow conditions," J. Rheol. 57, 1411-1428 (2013).

${ }^{74} \mathrm{Li}$, Y., Hu, M., McKenna, G. B., Dimitriou, C. J., McKinley, G. H. Mick, R. M., Venerus, D. C., and Archer, L. A., "Response to: Sufficiently entangled polymers do show shear strain localization at high enough weissenberg numbers," J. Rheol. 58, 1071-1082 (2014)

${ }^{75}$ Likhtman, A. E. and Graham, R. S., "Simple constitutive equation for linear polymer melts derived from molecular theory: Rolie-poly equation," JNNFM 114, 1 - 12 (2003).

${ }^{76}$ Liu, C., Ferrero, E. E., Martens, K., and Barrat, J.-L., "Creep dynamics of athermal amorphous materials: a mesoscopic approach," Soft Matter 14, 8306-8316 (2018)

${ }^{77}$ Mair, R. W. and Callaghan, P. T., "Shear flow of wormlike micelles in pipe and cylindrical couette geometries as studied by nuclear magnetic resonance microscopy," J. Rheol. 41, 901-923 (1997).
${ }^{78}$ Manneville, S., "Recent experimental probes of shear banding," Rheol. Acta 47, 301-318 (2008).

${ }^{79}$ Manneville, S., Colin, A., Waton, G., and Schosseler, F., "Wall slip, shear banding, and instability in the flow of a triblock copolymer micellar solution," Phys. Rev. E 75, 061502 (2007).

${ }^{80}$ Mansard, V., Colin, A., Chauduri, P., and Bocquet, L., "A kinetic elasto-plastic model exhibiting viscosity bifurcation in soft glassy materials," Soft Matter 7, 5524-5527 (2011).

${ }^{81}$ Martens, K., Bocquet, L., and Barrat, J.-L., "Spontaneous formation of permanent shear bands in a mesoscopic model of flowing disordered matter," Soft Matter 8, 4197-4205 (2012).

${ }^{82}$ Martin, J. and $\mathrm{Hu}, \mathrm{Y} .$, "Transient and steady-state shear banding in aging soft glassy materials," Soft Matter 8, 6940-6949 (2012).

${ }^{83}$ Milner, S. T., "Square patterns and secondary instability in driven capillary waves," J. Fluid Mech. 225, 81-100 (1991)

${ }^{84}$ Mohammadigoushki, H., Dalili, A., Zhou, L., and Cook, P., “Transient evolution of flow profiles in a shear banding wormlike micellar solution: experimental results and a comparison with the $\mathrm{vcm}$ model," Soft Matter 15, 5483-5494 (2019).

${ }^{85}$ Moorcroft, R., Cates, M., and Fielding, S., "Age dependent transient shear banding in soft glasses," Phys. Rev. Lett. 106, 055502 (2011)

${ }^{86}$ Moorcroft, R. and Fielding, S., "Criteria for shear banding in timedependent flows of complex fluids," Phys. Rev. Lett. 110, 086001 (2013)

${ }^{87}$ Moorcroft, R. L. and Fielding, S. M., "Shear banding in timedependent flows of polymers and wormlike micelles," J. Rheol. 58, 103-147 (2014), https://doi.org/10.1122/1.4842155.

${ }^{88}$ Nicolas, A., Ferrero, E. E., Martens, K., and Barrat, J.-L., “Deformation and flow of amorphous solids: Insights from elastoplastic models," Rev. Mod. Phys. 90, 045006 (2018).

${ }^{89}$ Olmsted, P. D., "Perspectives on shear banding in complex fluids," Rheol. Acta 47, 283-300 (2008).

${ }^{90}$ Olmsted, P. D., Radulescu, O., and Lu, C.-Y. D., "Johnson-segalman model with a diffusion term in cylindrical couette flow," J. Rheol. 44, 257-275 (2000)

${ }^{91}$ Paredes, J., Shahidzadeh-Bonn, N., and Bonn, D., "Shear banding in thixotropic and normal emulsions," J. Phys.: Condens. Matter 23, 284116 (2011)

${ }^{92}$ Perge, C., Fardin, M.-A., and Manneville, S., "Surfactant micelles: model systems for flow instabilities of complex fluids," Eur. Phys. J. E 37, 23-34 (2014)

${ }^{93}$ Peterson, J. D. and Cates, M. E., "A full-chain tube-based constitutive model for living linear polymers," Journal of Rheology 64, 14651496 (2020), https://doi.org/10.1122/8.0000114

${ }^{94}$ Peterson, J. D. and Cates, M. E., "Constitutive models for wellentangled living polymers beyond the fast-breaking limit," Journal of Rheology 65, 633-662 (2021), https://doi.org/10.1122/8.0000199.

${ }^{95}$ Picard, G., Ajdari, A., Bocquet, L., and Lequeux, F., "A simple model for heterogeneous flows of yield stress fluids," Phys. Rev. E 66, 051501 (2002).

${ }^{96}$ Pipe, C. J., Kim, N. J., Vasquez, P. A., Cook, L. P., and McKinley, G. H., "Wormlike micellar solutions: Ii. comparison between experimenta data and scission model predictions," Journal of Rheology 54, 881913 (2010), https://doi.org/10.1122/1.3439729.

${ }^{97}$ Ravindranath, S., Wang, S.-Q., Ofechnowicz, M., and Quirk, R. P., "Banding in simple steady shear of entangled polymer solutions," Macromolecules 41, 2663-2670 (2008).

${ }^{98}$ Rogers, S., Callaghan, P., Petekidis, G., and Vlassopoulos, D., "Timedependent rheology of colloidal star glasses," J. Rheol. 54, 133-158 (2010)

${ }^{99}$ Rogers, S. A., Vlassopoulos, D., and Callaghan, P. T., "Aging, yielding, and shear banding in soft colloidal glasses," Phys. Rev. Lett. 100, 128304 (2008)

${ }^{100}$ Salmon, J.-B., Colin, A., Manneville, S., and Molino, F., "Velocity profiles in shear-banding wormlike micelles," Phys. Rev. Lett. 90 228303 (2003)

${ }^{101}$ Salmon, J.-B., Manneville, S., and Colin, A., "Shear banding in a lyotropic lamellar phase. i. time-averaged velocity profiles," Phys. Rev. E 68, 051503 (2003)

${ }^{102}$ Salmon, J.-B., Manneville, S., and Colin, A., "Shear banding in a 
lyotropic lamellar phase. ii. temporal fluctuations," Phys. Rev. E 68, 051504 (2003).

${ }^{103}$ Schall, P. and van Hecke, M., "Shear bands in matter with granularity," Annu. Rev. Fluid Mech. 42, 67-88 (2010).

${ }^{104}$ Spenley, A., Cates, M. E., and McLeish, T. C. B., "Nonlinear rheology of wormlike micelles," Phys. Rev. Lett. 71, 939-942 (1993).

${ }^{105}$ Spenley, A., Yuan, X. F., and Cates, M. E., "Nonmonotonic constitutive laws and the formation of shear-banded flows," J. Phys. II France 6, 551-571 (1996).

${ }^{106}$ Tapadia, P. and Wang, S.-Q., "Direct visualization of continuous simple shear in non-newtonian polymeric fluids," Phys. Rev. Lett. 96, 016001 (2006)

${ }^{107}$ Vasisht, V. V., Roberts, G., and Del Gado, E., "Emergence and persistence of flow inhomogeneities in the yielding and fluidization of dense soft solids," Phys. Rev. E 102, 010604 (2020).

${ }^{108}$ Vasquez, P. A., McKinley, G. H., and Cook, L. P.,"A network scission model for wormlike micellar solutions - i. model formulation and viscometric flow predictions," J. Non-Newt. Fluid Mech. 144, 122139 (2007).

${ }^{109}$ Van der Walt, S., Schönberger, J. L., Nunez-Iglesias, J., Boulogne, F., Warner, J. D., Yager, N., Gouillart, E., and Yu, T., "scikit-image: image processing in python," PeerJ 2, e453 (2014).

${ }^{110}$ Wang, S.-Q., Liu, G., Cheng, S., Boukany, P. E., Wang, Y., and Li, X., "Letter to the editor: Sufficiently entangled polymers do show shear strain localization at high enough weissenberg numbers," J. Rheol. 58, 1059-1069 (2014).

${ }^{111}$ Wei, Y., Solomon, M. J., and Larson, R. G., “Time-dependent shear rate inhomogeneities and shear bands in a thixotropic yield-stress fluid under transient shear" Soft Matter 15, 7956-7967 (2019).

${ }^{112}$ Yao, N. Y., Larsen, R. J., and Weitz, D. A., "Probing nonlinear rheology with inertio-elastic oscillations," J. Rheol. 52, 1013-1025 (2008), times Cited: 17

${ }^{113}$ Yerushalmi, J., Katz, S., and Shinnar, R., "Stability of steady shear flows of some viscoelastic fluids," Chemical Engineering Science 25, 1891 (1970).

${ }^{114}$ Zhou, L., McKinley, G. H., and Cook, L. P., “Wormlike micellar solutions: Iii. vcm model predictions in steady and transient shearing flows," JNNFM 211, 70 - 83 (2014).

${ }^{115}$ Zhou, L., Vasquez, P. A., Cook, L. P., and McKinley, G. H., "Modeling the inhomogeneous response and formation of shear bands in steady and transient flows of entangled liquids," J. Rheol. 52, 591-623 (2008). 
(a)

(b)
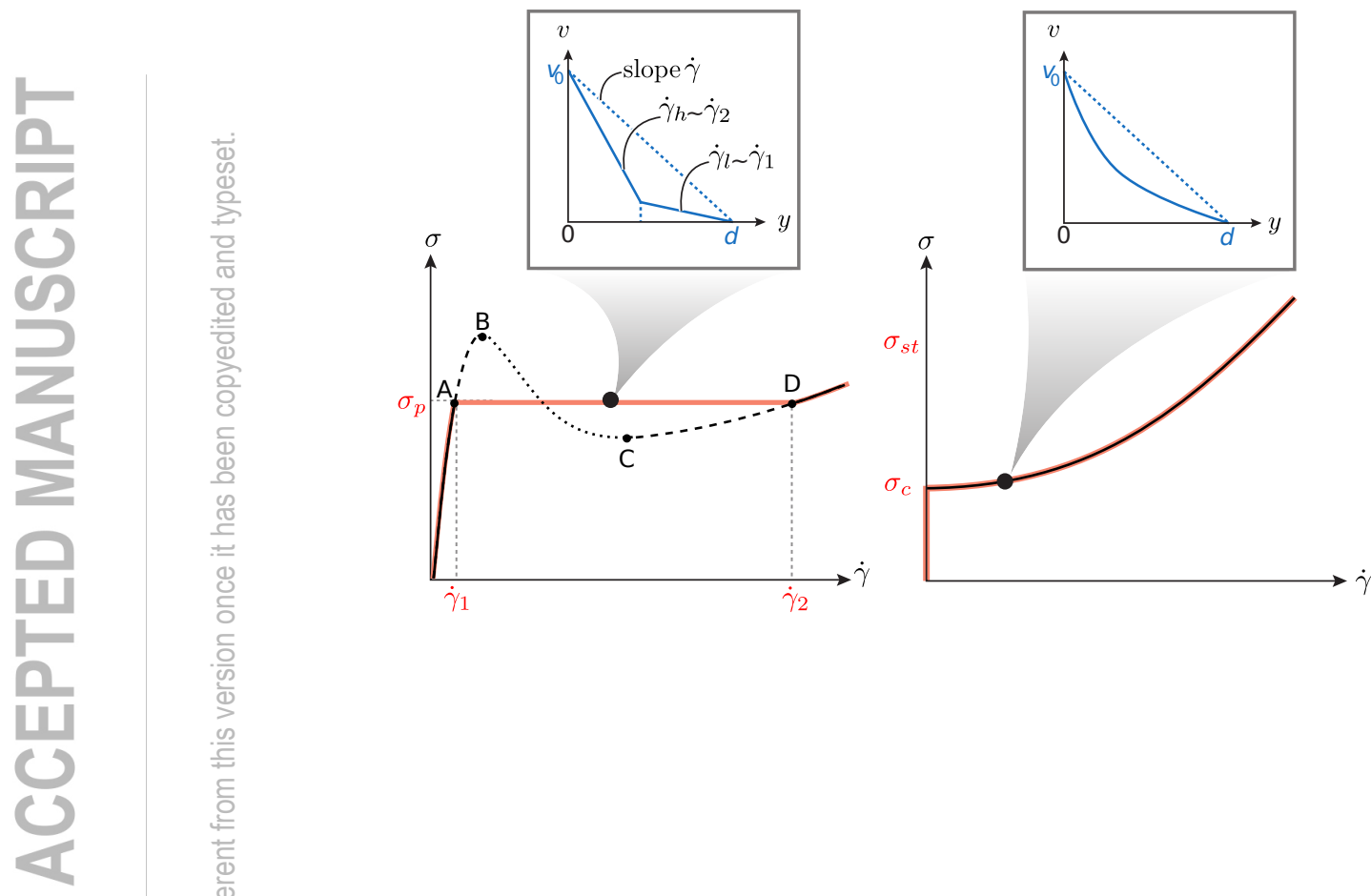

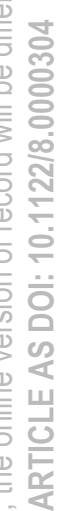

을

우 땅

능

嵌

空

焉

응

5

De 
(a)

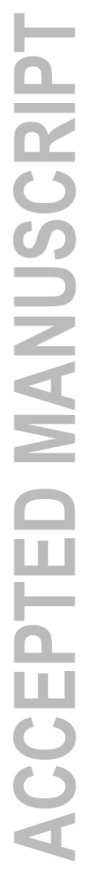

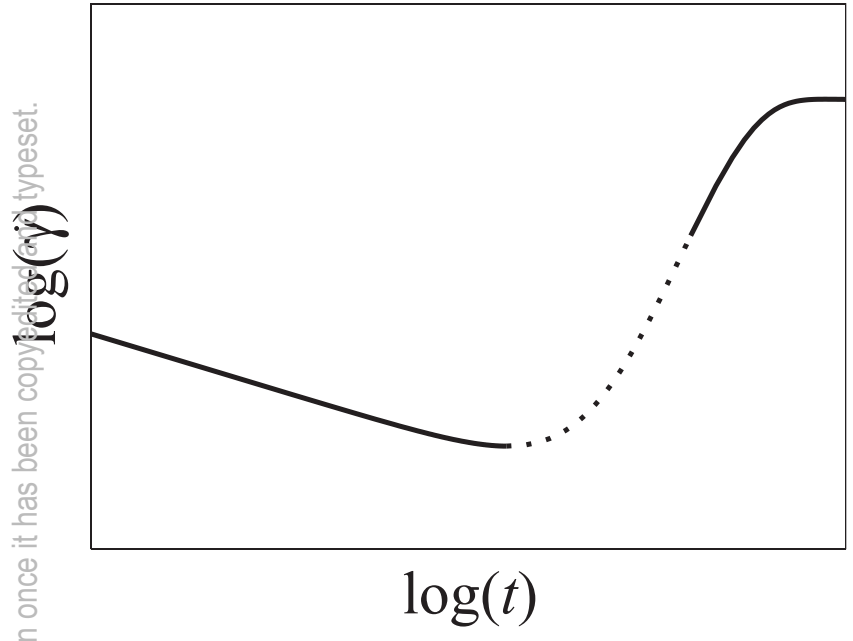

(b)

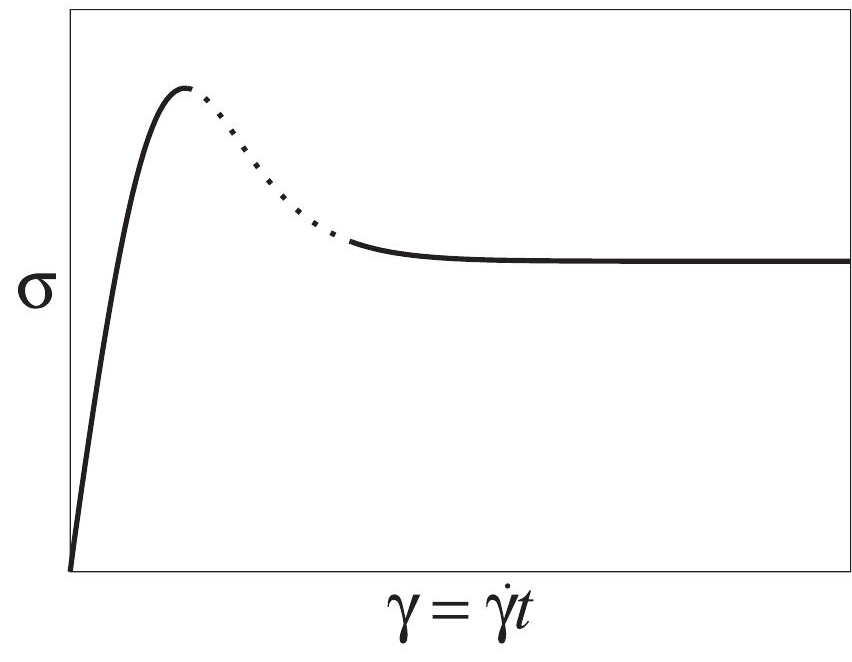




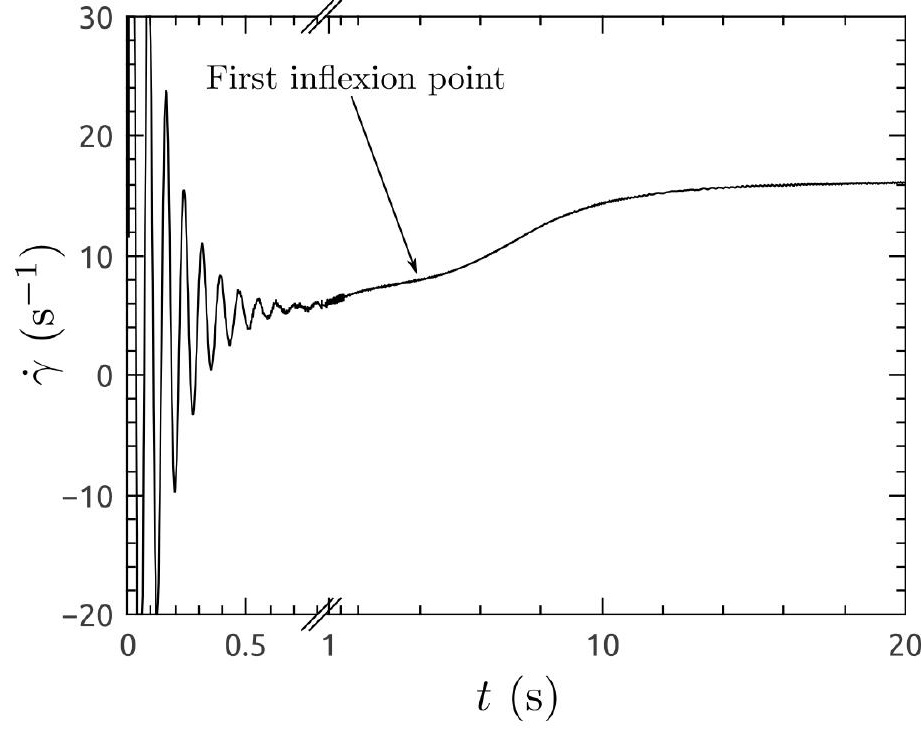




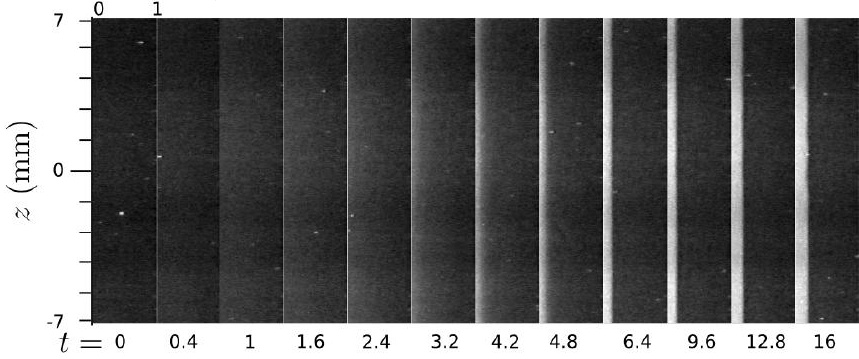

(b) $\dot{\gamma}=23 \mathrm{~s}^{-1}$
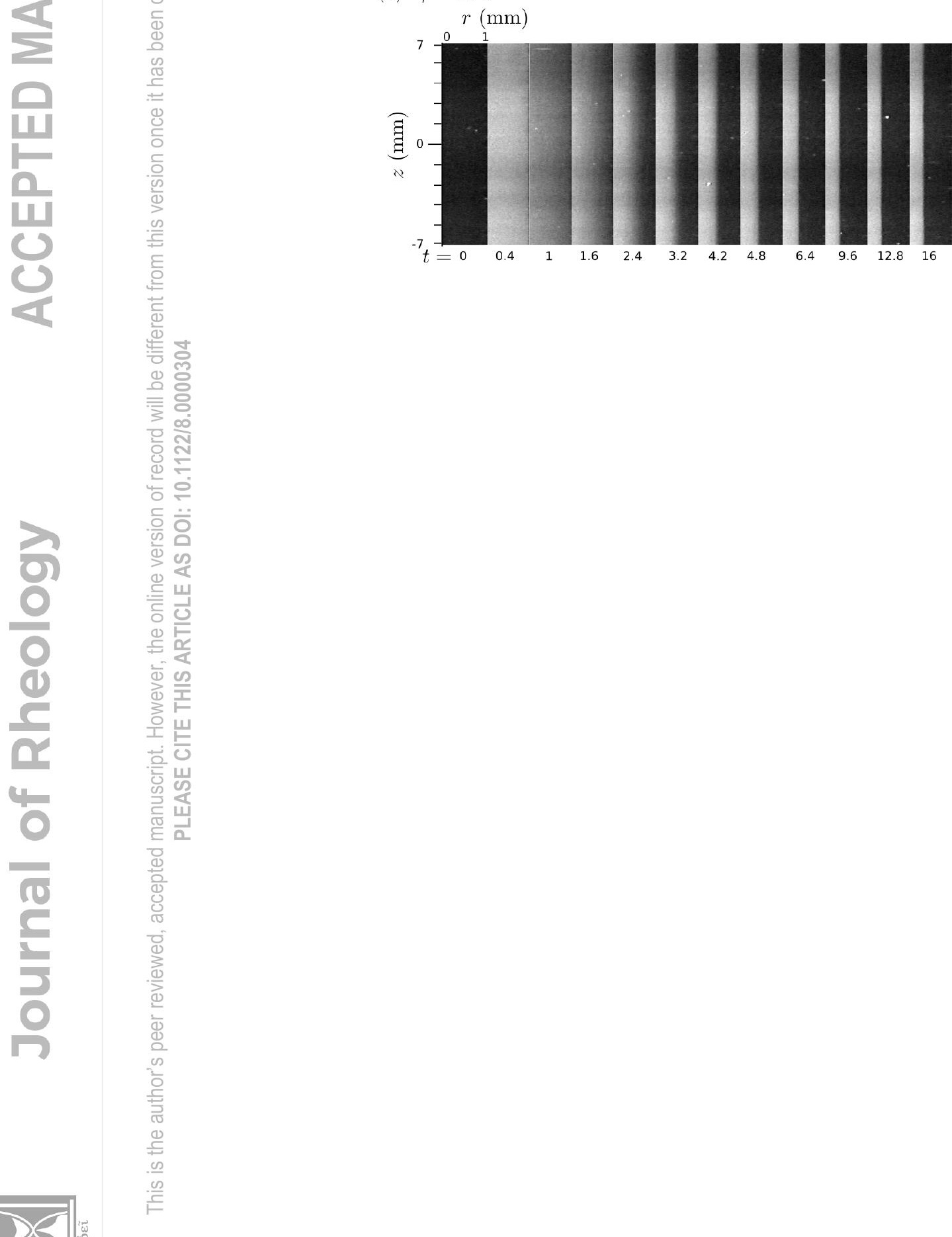
$\sigma=183 \mathrm{~Pa}$

(a)

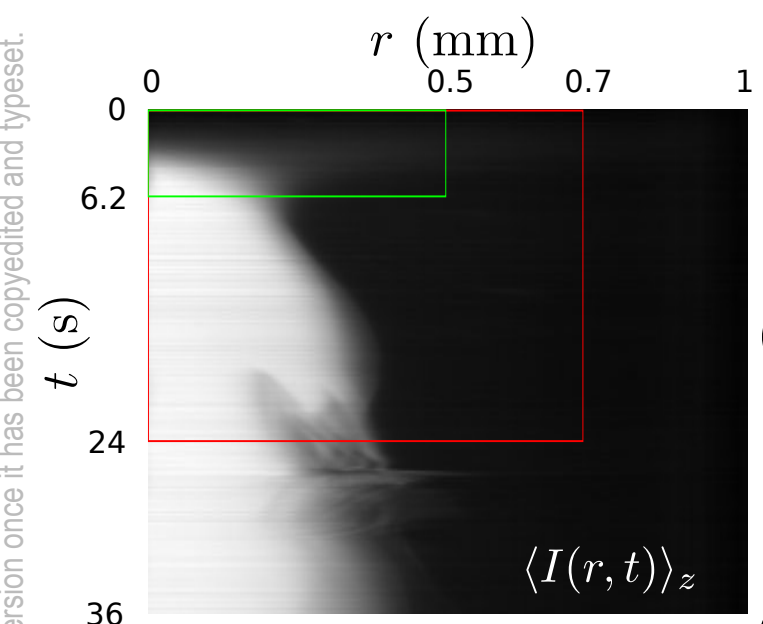

(b)

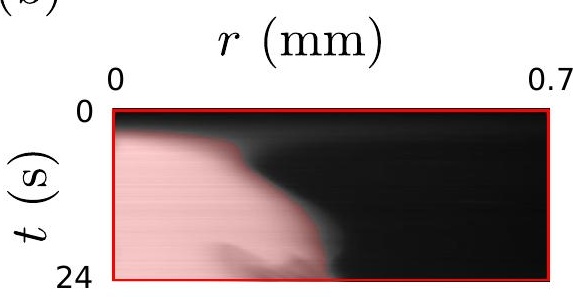

(c)

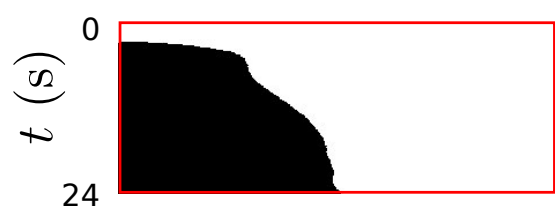

(d) $\quad r_{\text {front }}$

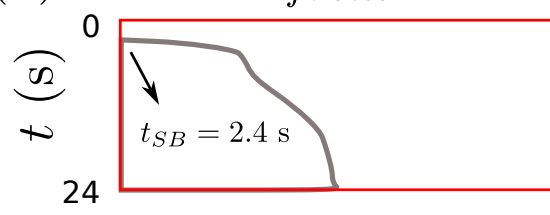

(e)

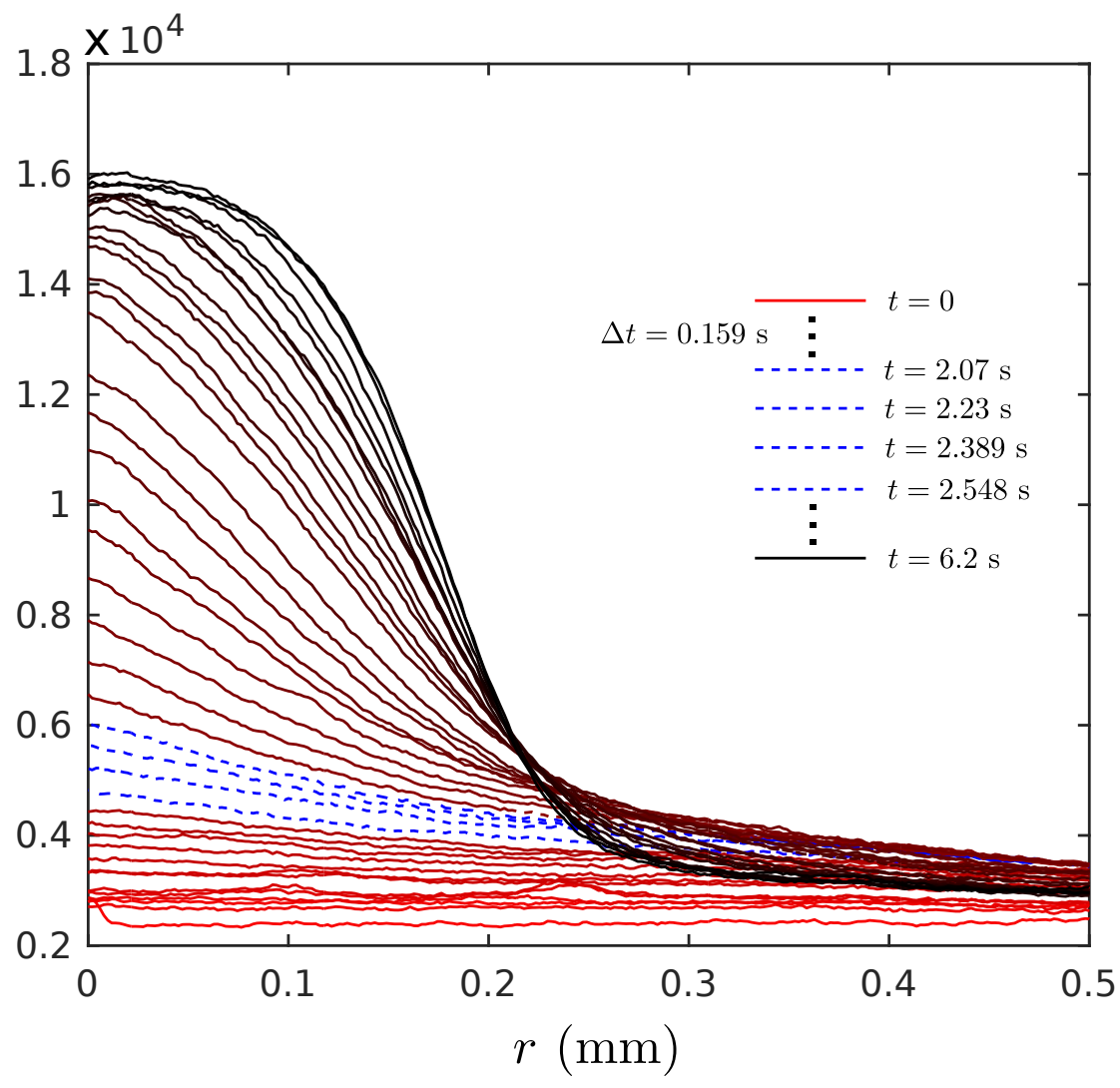



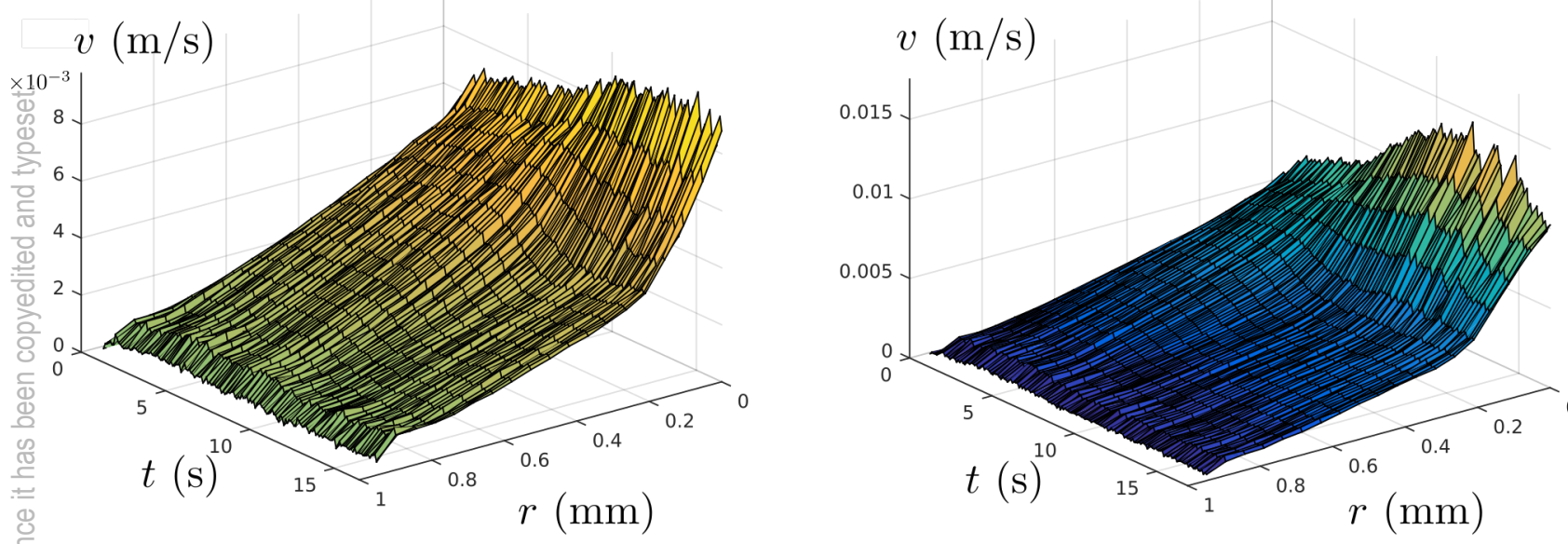

(c) $\sigma=183 \mathrm{~Pa}$

(d) $\sigma=188 \mathrm{~Pa}$
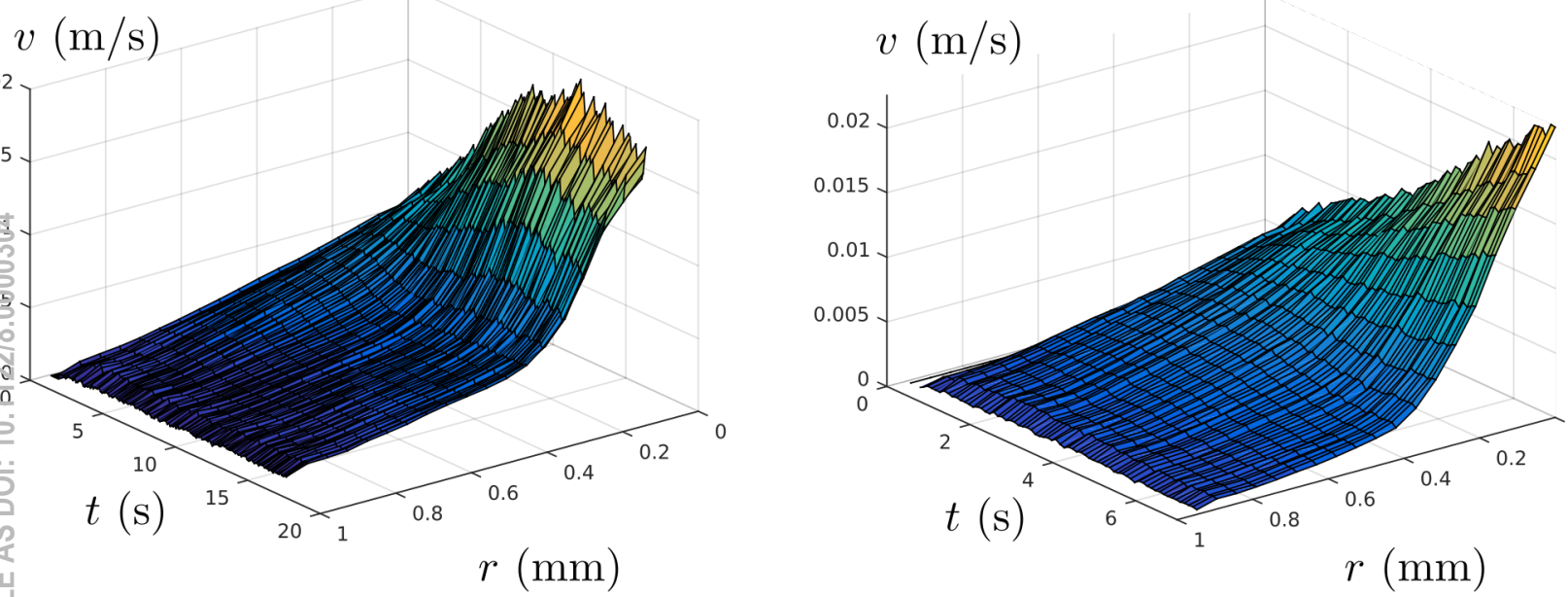

(e) $\sigma=183 \mathrm{~Pa}$
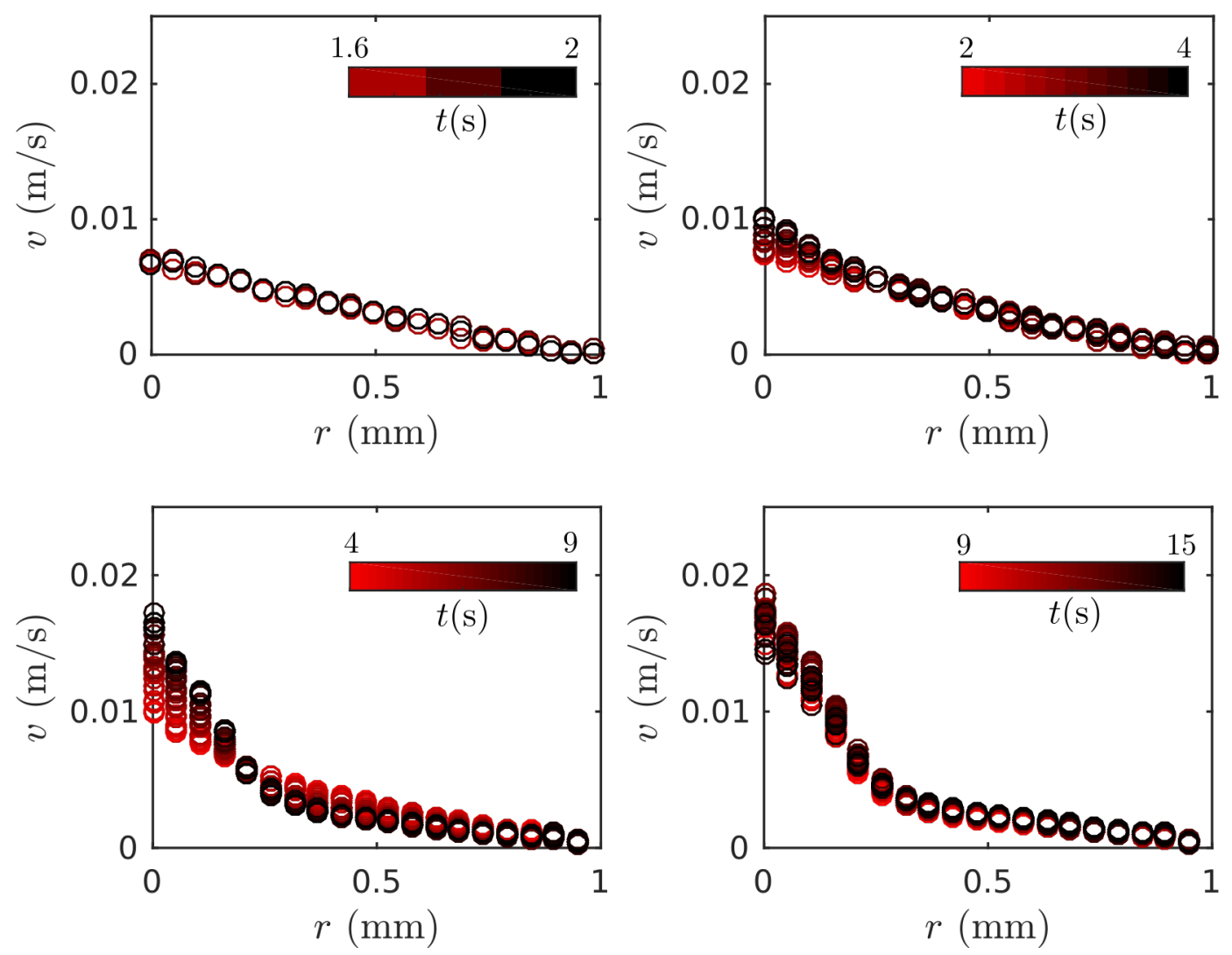
(a)

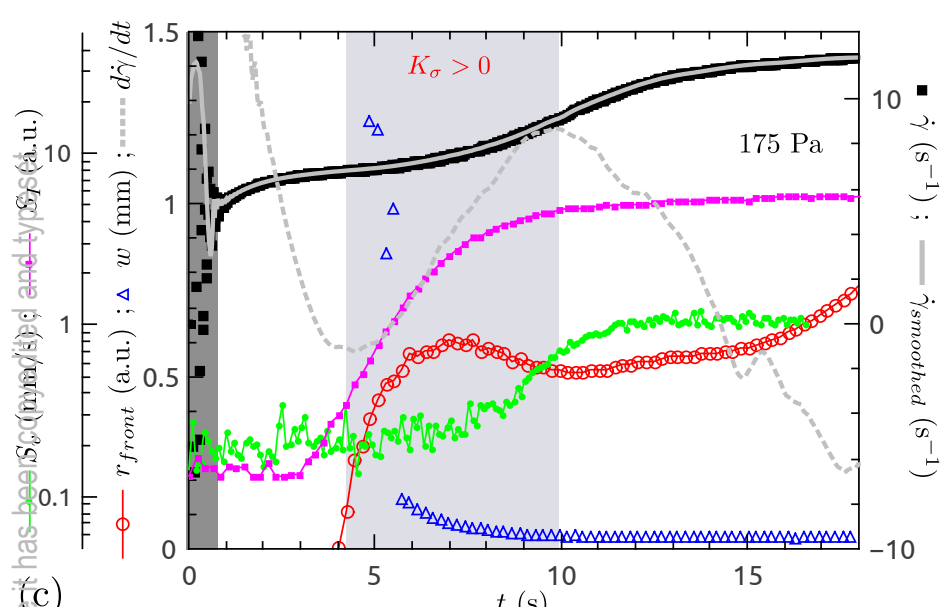

(c)

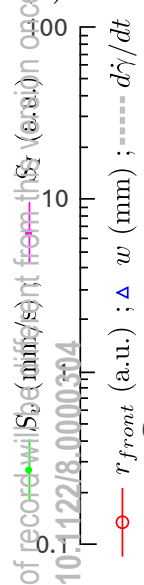

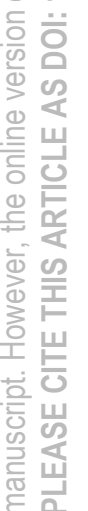

$t(\mathrm{~s})^{10}$

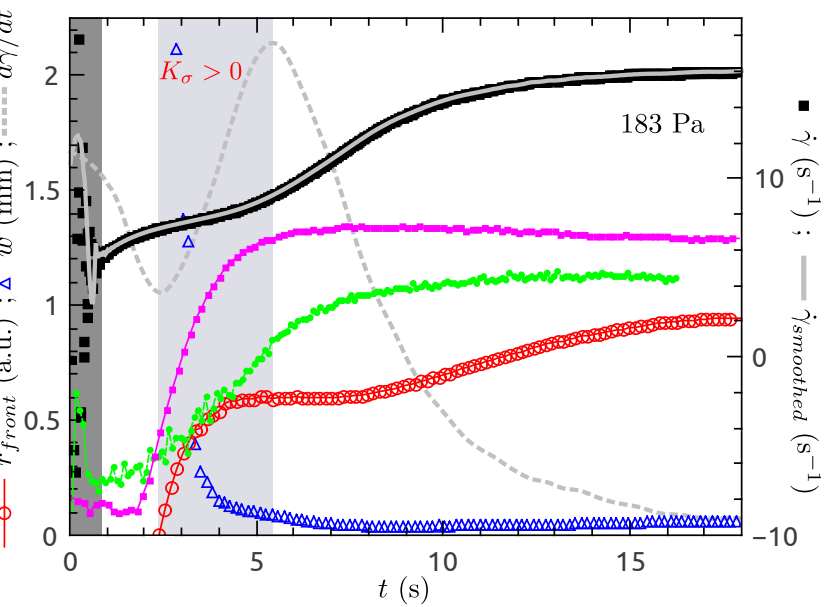

(d)

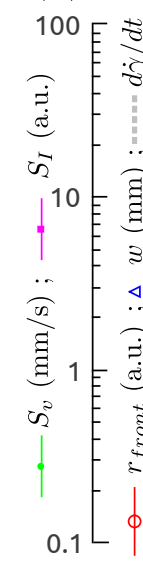

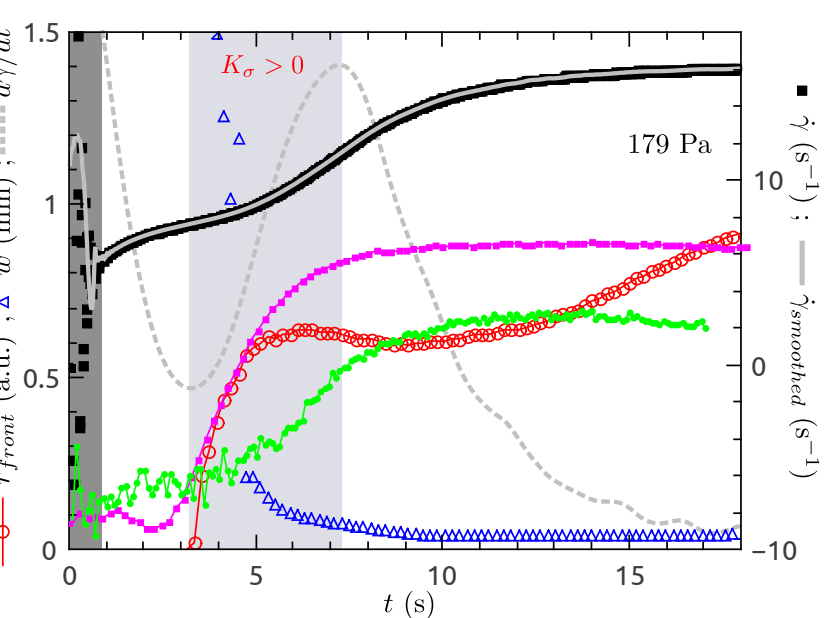

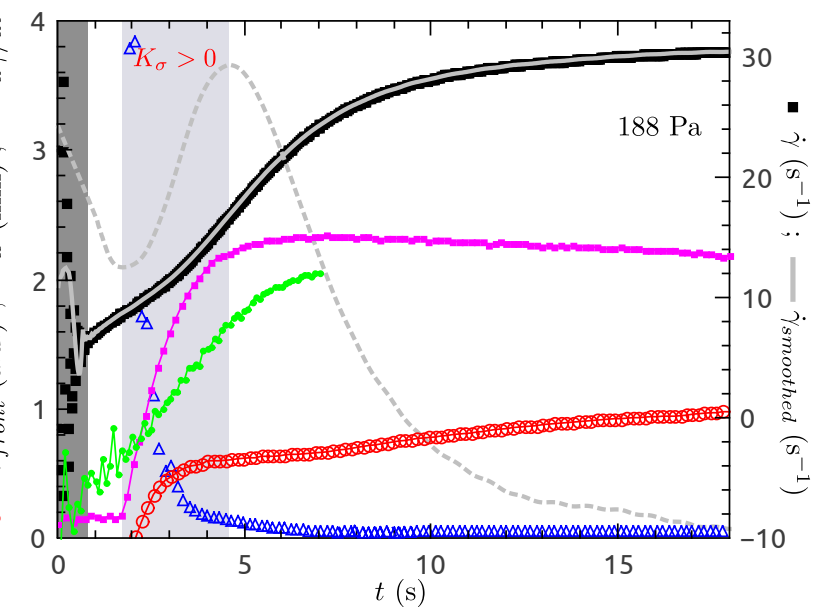


(a)

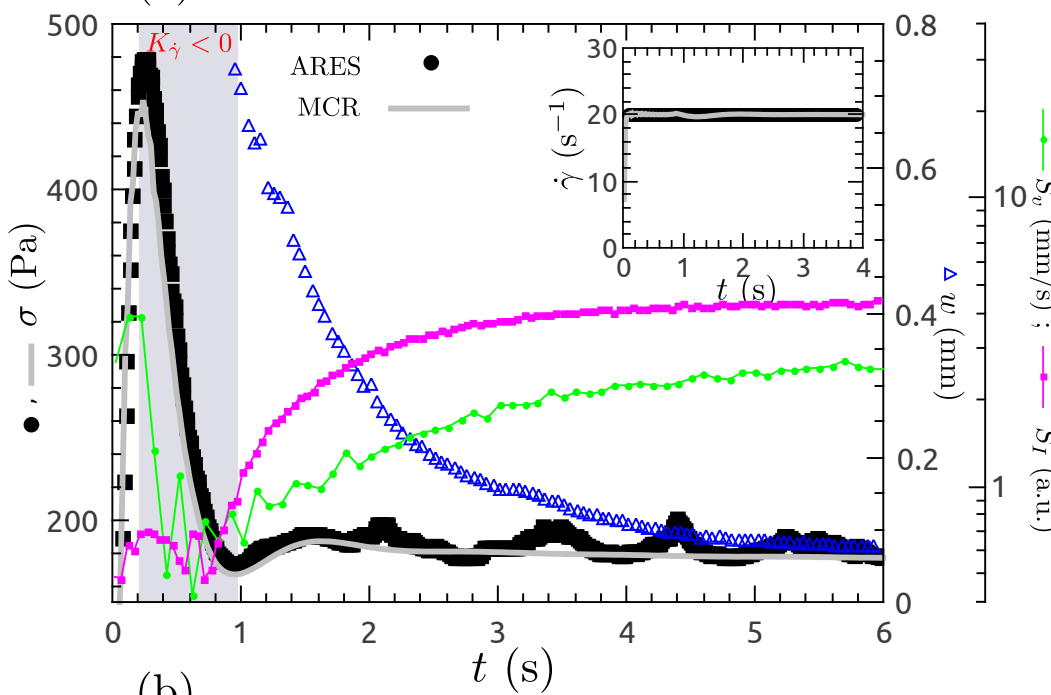

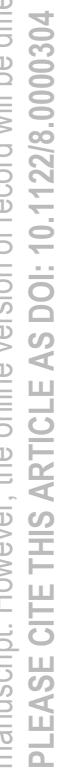
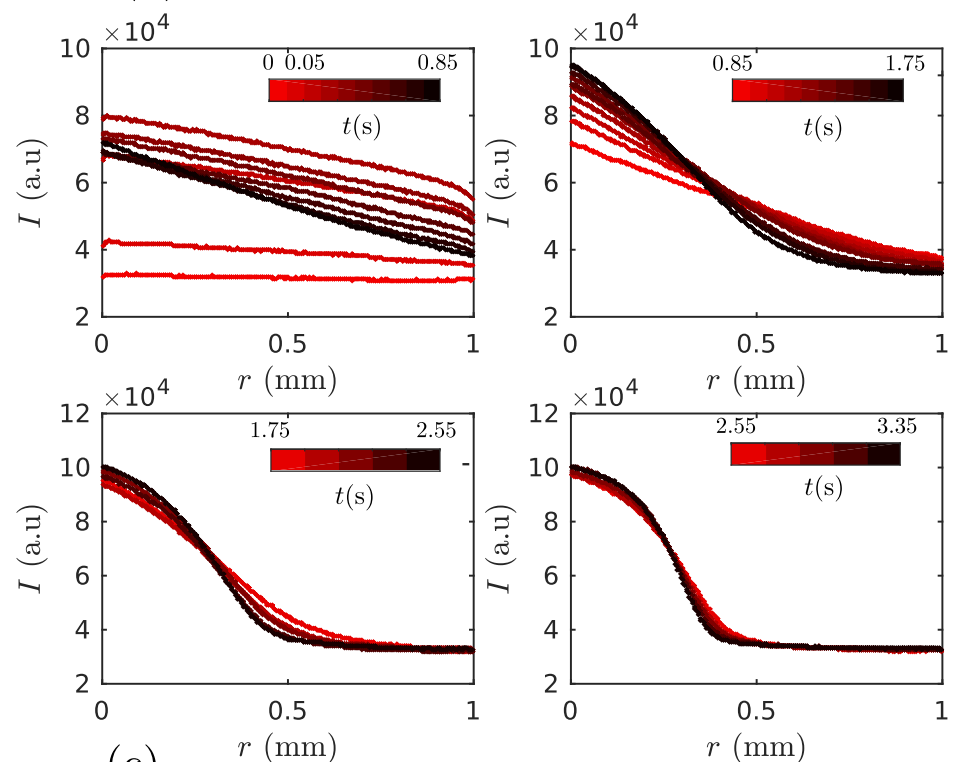

(c)
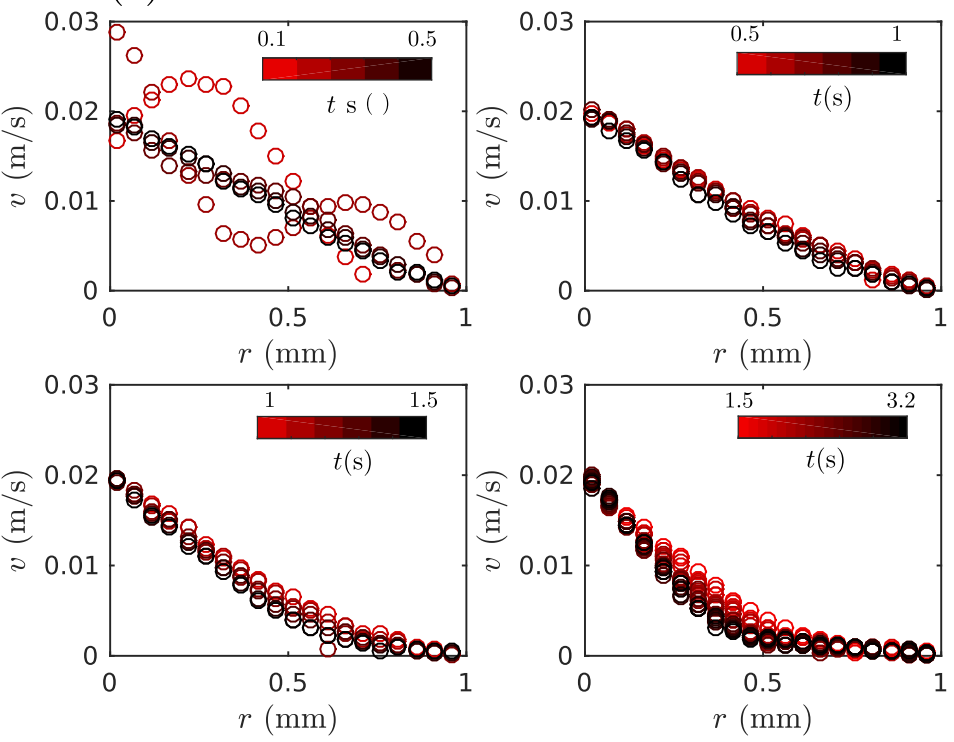
(a)

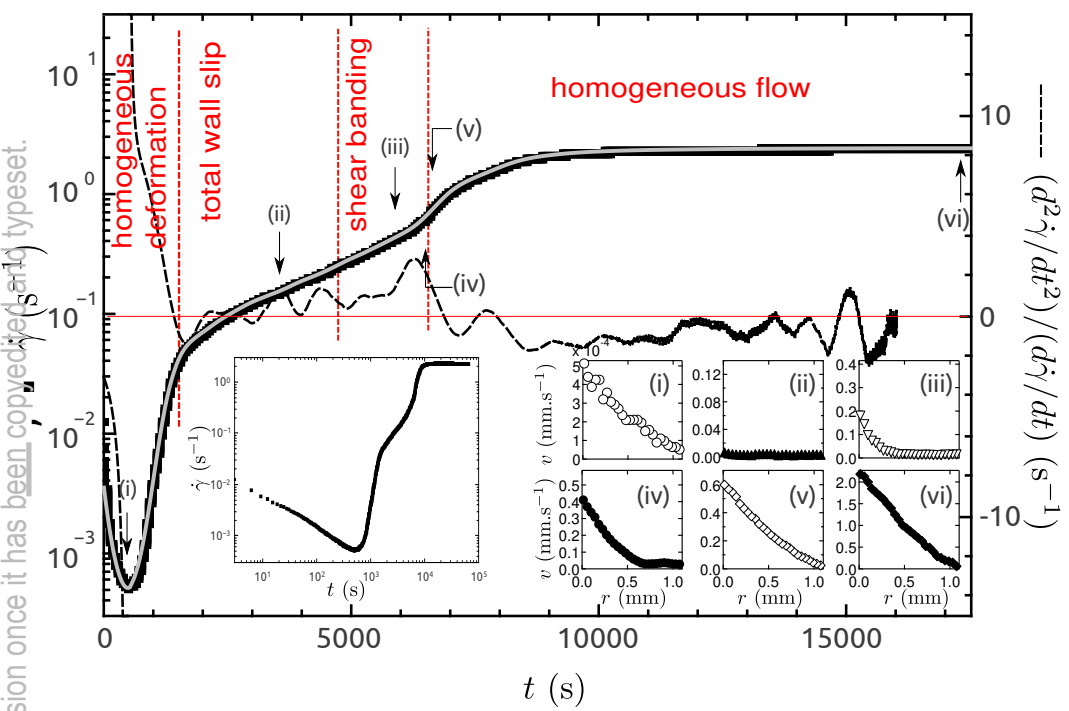

(b)

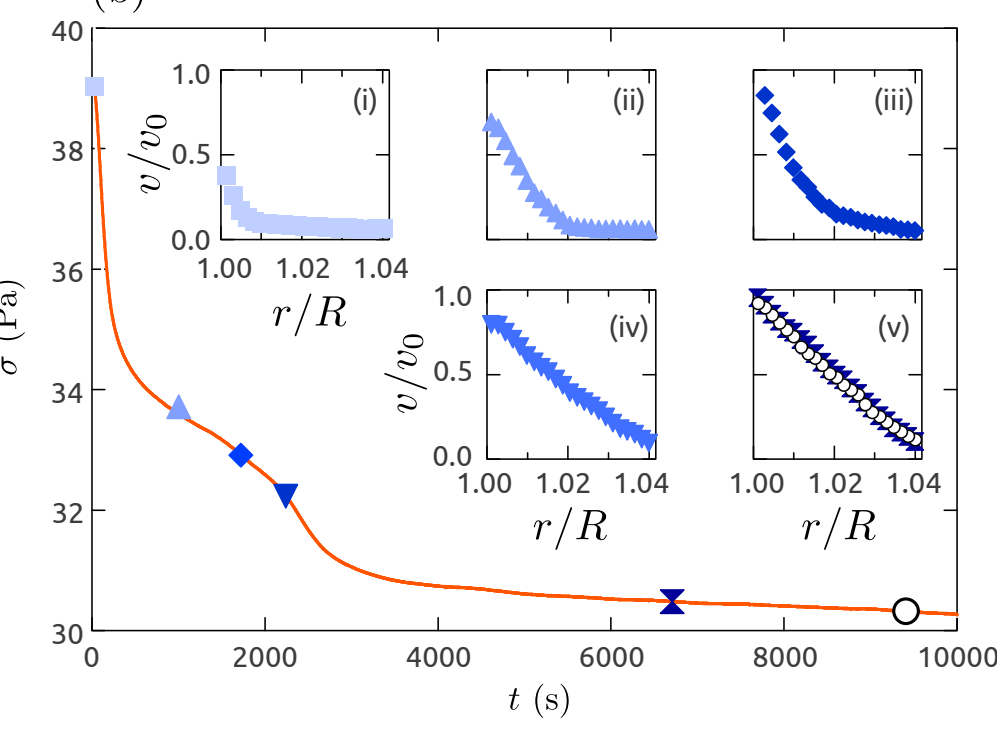




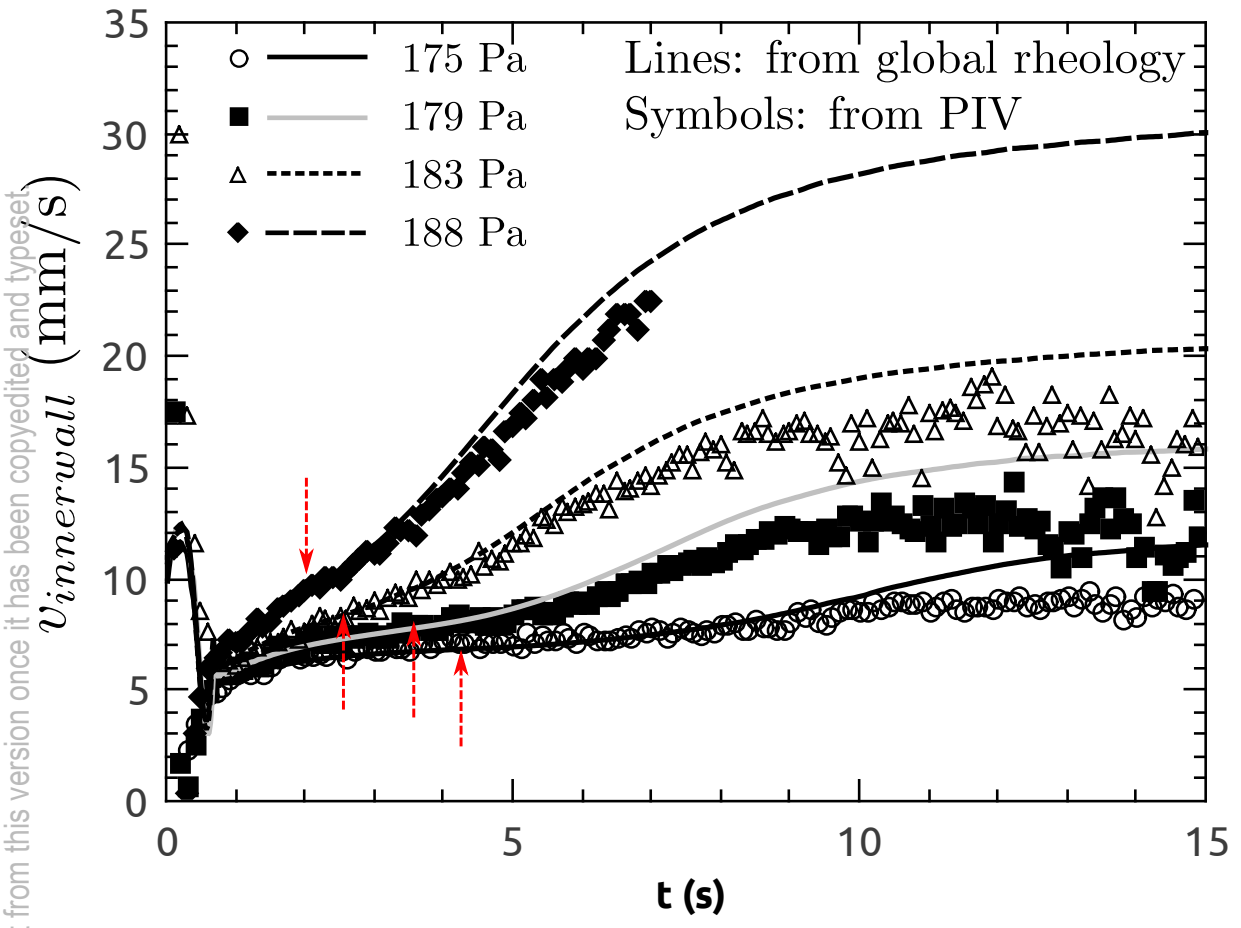



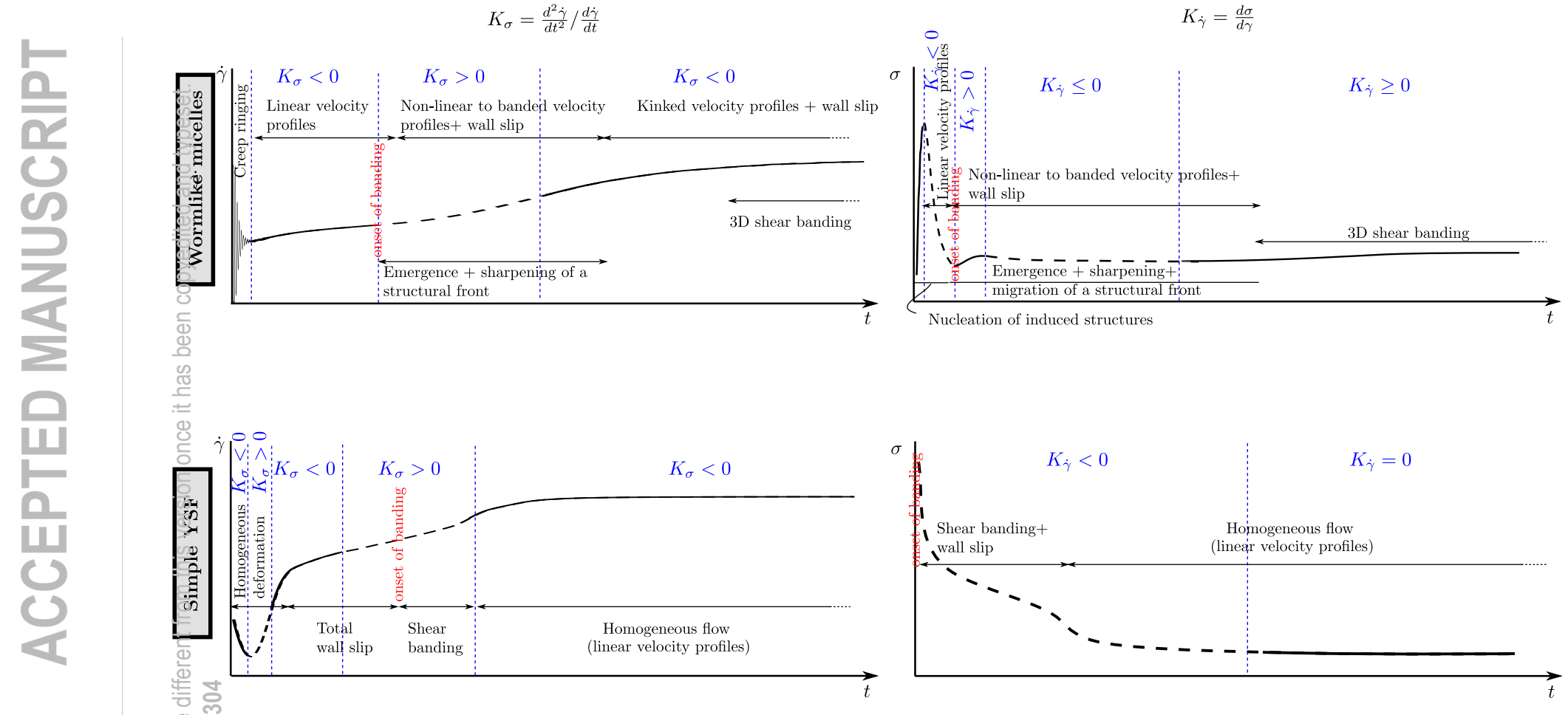